MATHEMATICS OF COMPUTATION

Volume 76, Number 257, January 2007, Pages 205-231

S $0025-5718(06) 01883-7$

Article electronically published on August 15, 2006

\title{
A SECOND-ORDER MAGNUS-TYPE INTEGRATOR FOR QUASI-LINEAR PARABOLIC PROBLEMS
}

\author{
C. GONZÁLEZ AND M. THALHAMMER
}

\begin{abstract}
In this paper, we consider an explicit exponential method of classical order two for the time discretisation of quasi-linear parabolic problems. The numerical scheme is based on a Magnus integrator and requires the evaluation of two exponentials per step. Our convergence analysis includes parabolic partial differential equations under a Dirichlet boundary condition and provides error estimates in Sobolev spaces. In an abstract formulation the initial boundary value problem is written as an initial value problem on a Banach space $X$

$$
u^{\prime}(t)=A(u(t)) u(t), \quad 0<t \leq T, \quad u(0) \text { given, }
$$

involving the sectorial operator $A(v): D \rightarrow X$ with domain $D \subset X$ independent of $v \in V \subset X$. Under reasonable regularity requirements on the problem, we prove the stability of the numerical method and derive error estimates in the norm of certain intermediate spaces between $X$ and $D$. Various applications and a numerical experiment illustrate the theoretical results.
\end{abstract}

\section{INTRODUCTION}

In this paper, we are concerned with the numerical solution of initial value problems of the form

$$
u^{\prime}(t)=A(u(t)) u(t), \quad 0<t \leq T, \quad u(0) \text { given. }
$$

Our main interest is to study (1.1) in an abstract setting where $A(v): D \subset X \rightarrow X$ is a family of sectorial operators on a Banach space $X$ which is defined for elements $v \in V \subset X_{\gamma}$ in an open subset of some intermediate space $D \subset X_{\gamma} \subset X$. The scope of applications includes quasi-linear parabolic partial differential equations under a boundary condition of Dirichlet type which arise in the modelling of diffusion processes with state-dependent diffusivity and in the study of fluids in porous media.

In the present work, we pursue our convergence and stability analysis of Magnustype integrators for the time discretisation of nonautonomous parabolic problems [1, 26, 27 and study an explicit exponential integration scheme for abstract quasilinear problems (1.1). The numerical method considered relies on a second-order Magnus integrator and requires the evaluation of two exponentials at each step.

In the last few years, due to the progress of the art and the increasing potentiality for the efficient calculation of the matrix exponential in nondubious ways 22 . (see [10, 16] and references cited therein), numerical methods based on the Magnus

Received by the editor December 20, 2004 and, in revised form, September 30, 2005.

2000 Mathematics Subject Classification. Primary 35K55, 35K90,65L20, 65M12.

Key words and phrases. Quasi-linear parabolic problems, Magnus integrators, stability, convergence. 
expansion have received a lot of attention. This is confirmed by a variety of recent works; as a small selection we mention [5, 7, 17, 18, 19, 29. Following an approach studied by Magnus [21] for a linear system of nonautonomous ordinary differential equations

$$
y^{\prime}(t)=A(t) y(t), \quad y(0) \text { given, }
$$

the solution is represented by the exponential of a time-dependent matrix $\Omega$

$$
y(t)=\mathrm{e}^{\Omega(t)} y(0), \quad t \geq 0,
$$

which is given by an infinite series of iterated integrals involving matrix commutators of $A$

$$
\Omega(t)=\int_{0}^{t} A(\tau) \mathrm{d} \tau-\frac{1}{2} \int_{0}^{t}\left[\int_{0}^{\tau} A(\sigma) \mathrm{d} \sigma, A(\tau)\right] \mathrm{d} \tau+\cdots .
$$

In order to obtain a numerical approximation to the exact solution of (1.2), the Magnus expansion (1.3) is truncated and the integrals are determined by means of a quadrature formula. For instance, applying the midpoint rule to the first integral and omitting the remaining terms yields the second-order approximation

$$
y_{1}=\mathrm{e}^{h A(h / 2)} y_{0}
$$

to the exact solution value at time $h>0$. Here, the numerical starting value $y_{0}$ is a suitable approximation to the exact initial value $y(0)$. Such interpolatory Magnus integrators were considered, e.g., by Iserles and Nørsett in [18, in the context of geometric integration, and, as proven by Hochbruck and Lubich in [17. This method class is also eminently suited for the time integration of spatial discretisations of time-dependent Schrödinger-type equations. In [11, 26, the second-order Magnus-type integrator (1.4) was studied for abstract parabolic problems and further extended to linear and semilinear equations.

The above considerations motivate the following Magnus-type integrator for differential equations of the form (1.1). For some initial value $u_{0} \approx u(0)$ and a stepsize $h>0$, the numerical solution $u_{1}$ is determined by the relation

$$
u_{1}=\mathrm{e}^{h A\left(U_{01}\right)} u_{0} \approx u(h) .
$$

As auxiliary approximation to the exact solution value at the midpoint of the interval $[0, h]$, the additional internal stage $U_{01}$ is calculated by means of a firstorder integrator

$$
U_{01}=\mathrm{e}^{h / 2 A\left(u_{0}\right)} u_{0} \approx u(h / 2) .
$$

By Taylor series expansions it is straightforward to show that this scheme has classical order 2. It is notable that (1.5) can also be considered as a Runge-Kutta Munthe-Kaas method.

The objective of the present work is to analyse the stability and convergence behaviour of the numerical method (1.5) in the situation where (1.1) constitutes a quasi-linear parabolic initial boundary value problem written as an initial value problem on a Banach space.

Our paper is organised as follows. In Section 2, we state the fundamental hypotheses on the differential equation in (1.1), and we further specify several applications that can be cast into our abstract setting. In Section [3, we introduce the Magnus-type integrator whose favourable stability and convergence properties in 
connection with parabolic problems are analysed in detail in the subsequent Sections 4 and 5. In particular, under reasonable regularity requirements on the data and the solution of the initial value problem (1.1), we state an error estimate in the norm of a certain intermediate space between the underlying Banach space $X$ and the domain $D$. In Section 6, we finally comment on an extension of the Magnustype integrator to equations with an additional inhomogeneity and illustrate the theoretical result by a numerical example.

\section{Problem Class and applications}

In this section, we state the fundamental assumptions on the problem class considered and illustrate the abstract framework by several applications. The hypotheses on the initial value problem (1.1) primarily rely on González \& Palencia 13 where Runge-Kutta time discretisations for quasi-linear parabolic problems were studied. However, in our notation we follow Lunardi [20] and the previous works [11, 26. For an extensive treatise of quasi-linear evolution equations, we refer to the works of Amann [1]-4]. The theory of sectorial operators and analytic semigroups is described in detail in the monographs [15, 20, 25]. A comprehensive overview of interpolation theory is given in [20; see also [6, 28.

To simplify the notation, we henceforth do not distinguish the arising constants. Thus, the positive quantities $K, L, M>0$ and $C>0$ possibly have different values at different occurrences.

2.1. Quasi-linear equation. We consider a complex Banach space $\left(X,\|\cdot\|_{X}\right)$ and a dense subspace $\left(D,\|\cdot\|_{D}\right)$ that we assume to be continuously embedded in $X$. For $0<\mu<1$ we denote by $X_{\mu}$ some intermediate space between $X$ and $D$ such that the norm in $X_{\mu}$ fulfills the relation

$$
\|x\|_{X_{\mu}} \leq K\|x\|_{X}^{1-\mu}\|x\|_{D}^{\mu}, \quad x \in D
$$

with a constant $K>0$. Specifically, we set $X_{0}=X$ and $X_{1}=D$.

The right-hand side of the differential equation in (1.1) is defined by the map $A: V \rightarrow L(D, X)$ where $V \subset X_{\gamma}$ is an open subset of some intermediate space $X_{\gamma}$ with $0 \leq \gamma<1$. In view of applications, the requirement that the domain of the unbounded linear operator $A(v): D \rightarrow X$ is independent of $v \in V$ implies that in general only initial boundary value problems involving a boundary condition of Dirichlet type are covered by our analysis. The fundamental assumptions on $A$ are as follows.

Hypothesis 2.1. (i) The closed linear operator $A(v): D \rightarrow X$ is uniformly sectorial for $v \in V$. Thus, there exist constants $a \in \mathbb{R}, 0<\phi<\pi / 2$, and $M>0$ such that for every $v \in V$ and for any complex number $\lambda \in \mathbb{C}$ in the complement of the sector

$$
S_{\phi}(a)=\{z \in \mathbb{C}:|\arg (a-z)| \leq \phi\} \cup\{a\},
$$

the resolvent $(\lambda I-A(v))^{-1}: X \rightarrow X$ exists and further satisfies the estimate

$$
\left\|(\lambda I-A(v))^{-1}\right\|_{X \leftarrow X} \leq \frac{M}{|\lambda-a|}, \quad \lambda \in \mathbb{C} \backslash S_{\phi}(a) .
$$

(ii) The graph norm of $A(v)$ and the norm in $D$ are equivalent, i.e., for every $v \in V$ the following relation holds with a constant $K>0$ :

$$
K^{-1}\|x\|_{D} \leq\|x\|_{X}+\|A(v) x\|_{X} \leq K\|x\|_{D}, \quad x \in D .
$$


(iii) For some $0 \leq \vartheta<1$ the intermediate space $X_{1+\vartheta}$ between $D$ and the domain of $A(v)^{2}$ does not depend on $v \in V$. Moreover, the map $A: V \rightarrow L\left(X_{1+\vartheta}, X_{\vartheta}\right)$ is Lipschitz-continuous with respect to $v$; that is, the estimate

$$
\|A(v)-A(w)\|_{X_{\vartheta} \leftarrow X_{1+\vartheta}} \leq L\|v-w\|_{X_{\gamma}}, \quad v, w \in V
$$

is valid with a constant $L>0$.

By Hypothesis 2.1(ii), a suitable choice for the intermediate space $X_{\gamma}, 0 \leq \gamma<1$, is the real interpolation space or the intermediate Calderón space, whereas, due to the nonapplicability of Heinz's theorem, a fractional power space may depend on $A(v), v \in V \subset X_{\gamma}$.

Quasi-linear parabolic initial boundary value problems where the above assumptions hold true are specified below in Subsection 2.2

Remark 2.2. In the situation of Hypothesis 2.1 with $\vartheta=0$, the unique solvability of the abstract initial value problem (1.1) is ensured. Namely, it is shown in Amann [2] that the quasi-linear differential equation defines a semiflow in $X_{\beta} \cap V$ for every $\gamma<\beta<1$. However, the limiting case $\beta=\gamma$ is not covered by this result.

We note that for a linear operator $F: X \rightarrow D$, relation (2.2) implies the bounds

$$
\|A(v) F\|_{X \leftarrow X} \leq K\|F\|_{D \leftarrow X}, \quad\|F\|_{D \leftarrow X} \leq K\left(1+\|A(v) F\|_{X \leftarrow X}\right) .
$$

Besides, after possibly enlarging the constant $M>0$, the following extension of the resolvent estimate (2.1) is valid:

$$
\left\|t^{\nu-\mu}(\lambda I-t A(v))^{-1}\right\|_{X_{\nu} \leftarrow X_{\mu}} \leq \frac{M}{|\lambda-a t|}, \quad t>0, \quad 0 \leq \mu \leq \nu \leq 1
$$

see also 12 . For any fixed $v \in V$ the sectorial operator $A(v): D \rightarrow X$ is the infinitesimal generator of an analytic semigroup $\left(\mathrm{e}^{t A(v)}\right)_{t \geq 0}$ on $X$. Here, the linear operator

$$
\mathrm{e}^{t A(v)}=\frac{1}{2 \pi \mathrm{i}} \int_{\Gamma} \mathrm{e}^{\lambda}(\lambda I-t A(v))^{-1} \mathrm{~d} \lambda, \quad t>0,
$$

is defined through the integral formula of Cauchy, where $\Gamma$ denotes a path that surrounds the spectrum of $A(v)$. If $t=0$, let $\mathrm{e}^{t A(v)}=I$. Therefore, due to (2.4), the estimates

$$
\begin{gathered}
\left\|t^{\nu-\mu} \mathrm{e}^{t A(v)}\right\|_{X_{\nu} \leftarrow X_{\mu}} \leq M, \quad 0 \leq t \leq T, \quad 0 \leq \mu \leq \nu \leq 1, \\
\left\|t^{1+\nu-\mu} A(v) \mathrm{e}^{t A(v)}\right\|_{X_{\nu} \leftarrow X_{\mu}} \leq M, \quad 0 \leq t \leq T, \quad 0 \leq \mu, \nu \leq 1,
\end{gathered}
$$

are valid; see also [20, Chapter 2]. Consequently, by means of the identity

we obtain the bound

$$
\mathrm{e}^{t A(v)}-I=\int_{0}^{t} A(v) \mathrm{e}^{\tau A(v)} \mathrm{d} \tau
$$

$$
\left\|\mathrm{e}^{t A(v)}-I\right\|_{X_{\nu} \leftarrow X_{\mu}} \leq M t^{-\nu+\mu}, \quad t>0, \quad 0 \leq \mu, \nu \leq 1
$$

For later use, we further introduce the bounded linear operators,

$$
\begin{gathered}
\varphi(t A(v))=\frac{1}{t} \int_{0}^{t} \mathrm{e}^{(t-\tau) A(v)} \mathrm{d} \tau, \quad t>0, \quad \varphi(t A(v))=I, \quad t=0, \\
\psi(t A(v))=\frac{1}{t^{2}} \int_{0}^{t} \tau \mathrm{e}^{(t-\tau) A(v)} \mathrm{d} \tau, \quad t>0, \quad \psi(t A(v))=\frac{1}{2} I, \quad t=0,
\end{gathered}
$$


which are related to the analytic semigroup. Moreover, with the help of the integral formula of Cauchy, the validity of the relation

$$
\psi(t A(v))-1 / 2 \varphi(t A(v))=t A(v) \chi(t A(v))
$$

with a bounded linear operator $\chi(t A(v))$ follows. More precisely, as a direct consequence of the defining relations and (2.6), we obtain the estimate

$$
\begin{aligned}
& \left\|t^{\nu-\mu} \varphi(t A(v))\right\|_{X_{\nu} \leftarrow X_{\mu}}+\left\|t^{\nu-\mu} \psi(t A(v))\right\|_{X_{\nu} \leftarrow X_{\mu}} \\
& \quad+\|\chi(t A(v))\|_{X_{\mu} \leftarrow X_{\mu}} \leq M, \quad 0 \leq t \leq T, \quad 0 \leq \mu \leq \nu \leq 1,
\end{aligned}
$$

with a constant $M>0$.

We close this subsection with some useful abbreviations. In the rest of the paper, the closed ball in $X_{\mu}$ with radius $\varrho>0$ and center $v^{*} \in X_{\mu}$ is denoted by

$$
B_{\mu}\left(v^{*}, \varrho\right)=\left\{v \in X_{\mu}:\left\|v-v^{*}\right\|_{X_{\mu}} \leq \varrho\right\} \subset X_{\mu} .
$$

Further, for a family $f=\left(f_{n}\right)_{0 \leq n \leq N}$ of bounded maps $f_{n}: I_{n} \subset \mathbb{R} \rightarrow X_{\mu}$ or for a sequence $g=\left(g_{n}\right)_{0 \leq n \leq N}$ in $X_{\mu}$, we set

$$
\begin{gathered}
\|f\|_{X_{\mu}, \infty}=\max _{0 \leq n \leq N}\left\|f_{n}\right\|_{X_{\mu}, \infty}, \quad\left\|f_{n}\right\|_{X_{\mu}, \infty}=\max _{t \in I_{n}}\left\|f_{n}(t)\right\|_{X_{\mu}}, \\
\|g\|_{X_{\mu}, \infty}=\max _{0 \leq n \leq N}\left\|g_{n}\right\|_{X_{\mu}} .
\end{gathered}
$$

2.2. Applications. The following initial boundary value problem can be cast into the abstract setting of Subsection [2.1, see also [13].

Example 2.3. Let $\Omega$ be an open and bounded domain in $\mathbb{R}^{d}$ with regular boundary $\partial \Omega$. We consider the following partial differential equation for a real-valued function $U: \Omega \times[0, T] \rightarrow \mathbb{R}:(x, t)=\left(x_{1}, x_{2}, \ldots, x_{d}, t\right) \rightarrow U(x, t)$

$$
\partial_{t} U(x, t)=\mathcal{A}(U(x, t)) U(x, t), \quad x \in \Omega, \quad 0<t \leq T,
$$

subject to a homogeneous Dirichlet boundary condition and an initial condition

$$
U(x, t)=0, \quad x \in \partial \Omega, \quad 0 \leq t \leq T, \quad U(x, 0)=U_{0}(x), \quad x \in \Omega .
$$

Here, for $v \in C^{1}(\Omega)$ and $w \in C^{2}(\Omega)$ the second-order differential operator $\mathcal{A}$ is defined through

$$
\mathcal{A}(v(x)) w(x)=\sum_{i, j=1}^{d} a_{i j}(x, v(x), \nabla v(x)) \partial_{x_{i} x_{j}} w(x), \quad x \in \Omega .
$$

We suppose that the real-valued coefficients $a_{i j}$, which are defined on an open domain $\Omega \times \Lambda \subset \mathbb{R}^{d} \times \mathbb{R} \times \mathbb{R}^{d}$, satisfy suitable regularity and boundedness assumptions, and we further impose the ellipticity condition

$$
\sum_{i, j=1}^{d} a_{i j}(x, p, q) \xi_{i} \xi_{j} \geq \kappa \sum_{i=1}^{d} \xi_{i}^{2}, \quad(x, p, q) \in \Omega \times \Lambda, \quad \xi \in \mathbb{R}^{d},
$$

for some $\kappa>0$.

By suppressing the spatial variable, the initial boundary value problem (2.11) takes the form of an abstract initial value problem (1.1) on the Banach space

$$
X=L^{p}(\Omega), \quad d<p<\infty .
$$


More precisely, we set $(u(t))(x)=U(x, t)$ and define the linear operator $A(v)$ through $(A(v) w)(x)=\mathcal{A}(v(x)) w(x)$. Then, by choosing

$$
D=W^{2, p}(\Omega) \cap W_{0}^{1, p}(\Omega), \quad V=X_{\gamma}, \quad 1 / 2+d(2 p)^{-1}<\gamma<1,
$$

it follows that Hypothesis 2.1 is satisfied with $\vartheta=0$. In particular, due to the imbedding $X_{\gamma} \subset C^{1}(\Omega)$, the linear operator $A(v): D \rightarrow X$ is well defined for elements $v \in X_{\gamma}$; see [15, Section 1.6]. If the coefficients of the differential operator do not depend on the derivative, the less restrictive condition $d(2 p)^{-1}<\gamma<1$ follows.

The following illustration describes the movement of a fluid of variable density through a porous medium under the influence of gravity and hydrodynamic dispersion. It is shown in Clément et al. 9] that the specified system of elliptic-parabolic partial differential equations when reformulated as an abstract evolution equation on a suitably chosen Banach space leads to a quasi-linear parabolic problem.

Example 2.4. Let $\Omega \subset \mathbb{R}^{2}$ be a rectangle or an open and bounded domain in $\mathbb{R}^{2}$ with regular boundary $\partial \Omega$. Elements $x=\left(x_{1}, x_{2}\right)^{T} \in \mathbb{R}^{2}$ are meanwhile interpreted as columns. We consider a system of elliptic-parabolic partial differential equations for functions $U, V: \Omega \times[0, T] \rightarrow \mathbb{R}:(x, t) \rightarrow U(x, t)$

$$
\left\{\begin{array}{l}
-\Delta V(x, t)=\partial_{x_{1}} U(x, t), \\
\partial_{t} U(x, t)+\operatorname{div} F(x, t)=0,
\end{array} \quad x \in \partial \Omega, \quad 0<t \leq T,\right.
$$

with map $F=\left(F_{1}, F_{2}\right)^{T}: \Omega \times[0, T] \rightarrow \mathbb{R}^{2}$ defined by

$$
F(x, t)=\operatorname{curl} V(x, t) U(x, t)-D(\operatorname{curl} V(x, t)) \nabla U(x, t) .
$$

Here, we set curl $V=\left(-\partial_{x_{2}} V, \partial_{x_{1}} V\right)^{T}$ and further employ the standard notations $\nabla U=\left(\partial_{x_{1}} U, \partial_{x_{2}} U\right)^{T}, \Delta V=\partial_{x_{1}}^{2} V+\partial_{x_{2}}^{2} V$, and $\operatorname{div} F=\partial_{x_{1}} F_{1}+\partial_{x_{2}} F_{2}$. The system (2.13a is subject to the boundary conditions

$$
V(x, t)=0, \quad \nu^{T} F(x, t)=0, \quad x \in \partial \Omega, \quad 0<t \leq T,
$$

where $\nu=\left(\nu_{1}, \nu_{2}\right)^{T}$ is the outward normal unit vector on $\partial \Omega$. Moreover, we impose a certain initial condition $U(x, 0)=U_{0}(x)$ for $x \in \Omega$. Specifically, the real-valued functions $D_{i j}: \mathbb{R}^{2} \rightarrow \mathbb{R}: q \rightarrow D(q)$ that define the hydrodynamic dispersion matrix $D(q)=\left(D_{i j}(q)\right)_{1 \leq i, j \leq 2}$ are given by

$$
D_{i j}(q)= \begin{cases}\left(c_{1}+c_{2} \sqrt{q_{1}^{2}+q_{2}^{2}}\right) \delta_{i j}+c_{3} \frac{q_{i} q_{j}}{\sqrt{q_{1}^{2}+q_{2}^{2}}}, & \text { if } q \neq 0, \\ c_{1} \delta_{i j}, & \text { if } q=0 .\end{cases}
$$

The positive constants $c_{1}, c_{2}$, and $c_{3}$ involve certain characteristic quantities such as the transversal and longitudinal dispersion length, the molecular diffusion coefficient, as well as the tortuosity and porosity of the medium. As usual, $\delta_{i j}$ denotes the Kronecker symbol. In particular, the ellipticity condition

$$
\sum_{i, j=1}^{2} D_{i j}(q) \xi_{i} \xi_{j} \geq \kappa\left(\xi_{1}^{2}+\xi_{2}^{2}\right), \quad q \in \mathbb{R}^{2}, \quad \xi \in \mathbb{R}^{d},
$$

holds for some $\kappa>0$.

Using the well-known result that the differential operator $-\Delta$ subject to a homogeneous Dirichlet boundary condition is invertible in $L^{p}(\Omega)$, we express the solution $V$ of (2.13a) in terms of $U$. That is, denoting the inverse operator by 
$(-\Delta \mid \gamma)^{-1}$, we get the relation $V=(-\Delta \mid \gamma)^{-1} \partial_{x_{1}} U$. Furthermore, by introducing the solution-dependent coefficients $a_{i}$ and $a_{i j}, 1 \leq i, j \leq 2$, such that

$$
\begin{gathered}
-\operatorname{curl} V(x, t)=-\operatorname{curl}\left((-\Delta \mid \gamma)^{-1} \partial_{x_{1}} U(x, t)\right)=\left(\begin{array}{l}
a_{1}(U(x, t)) \\
a_{2}(U(x, t))
\end{array}\right), \\
D(\operatorname{curl} V(x, t))=\left(\begin{array}{ll}
a_{11}(U(x, t)) & a_{12}(U(x, t)) \\
a_{21}(U(x, t)) & a_{22}(U(x, t))
\end{array}\right)
\end{gathered}
$$

problem (2.13) takes the form

$$
\partial_{t} U(x, t)=\mathcal{A}(U(x, t)) U(x, t), \quad x \in \Omega, \quad 0<t \leq T,
$$

with differential operator $\mathcal{A}$ given by

$$
\begin{aligned}
\mathcal{A}(U(x, t)) U(x, t)= & \sum_{i=1}^{2} \partial_{x_{i}} a_{i}(U(x, t)) U(x, t) \\
& +\sum_{i, j=1}^{2} \partial_{x_{i}} a_{i j}(U(x, t)) \partial_{x_{j}} U(x, t) .
\end{aligned}
$$

In addition, the solution $U$ fulfills the boundary condition

$$
\sum_{i=1}^{2} \nu_{i} a_{i}(U(x, t)) U(x, t)+\sum_{i, j=1}^{2} \nu_{i} a_{i j}(U(x, t)) \partial_{x_{j}} U(x, t)=0
$$

for $x \in \partial \Omega$ and $0<t \leq T$ as well as the initial condition $U(x, 0)=U_{0}(x)$ for $x \in \Omega$.

In order to cast this parabolic initial boundary value problem into our abstract framework, we set

$$
Y=W^{1, p^{\prime}}(\Omega), \quad X=Y^{\prime}, \quad D=W^{1, p}(\Omega)
$$

for $2<p<\infty$ and $1 / p^{\prime}=1-1 / p$. Besides, we define $A(u) u$ for $u \in D$ through

$$
\langle A(u) u, v\rangle=\sum_{i=1}^{2}\left\langle\partial_{x_{i}} v, a_{i}(u) u\right\rangle+\sum_{i, j=1}^{2}\left\langle\partial_{x_{i}} v, a_{i j}(u) \partial_{x_{j}} u\right\rangle, \quad v \in Y,
$$

where we employ the standard notation

$$
\langle f, g\rangle=\int_{\Omega} f(x) g(x) \mathrm{d} x, \quad f \in L^{p}(\Omega), \quad g \in L^{p^{\prime}}(\Omega) .
$$

In (2.15), due to the imbedding $W^{1, p}(\Omega) \subset C(\Omega)$, the coefficients $a_{i}(u)$ and $a_{i j}(u)$ are defined pointwise on the closure of $\Omega$. The investigations in [9] imply that the operator family $A: V \rightarrow L(D, X)$ satisfies Hypothesis 2.1 with $V=X_{\gamma}$ for $1 / 2+1 / p<\gamma<1$ and $\vartheta=0$.

\section{Magnus-Type InTEgRATOR}

In the rest of this paper, we specify the numerical scheme for the time discretisation of quasi-linear parabolic problems.

Henceforth, for integers $n \geq 0$ let $t_{n}=n h$ be the grid points associated with a constant stepsize $h>0$. The numerical approximation $u_{n+1}$ to the value of the 
exact solution of the abstract initial value problem (1.1) at time $t_{n+1}$ is determined through the recurrence formula

$$
\begin{gathered}
U_{n 1}=\mathrm{e}^{h / 2 A_{n}} u_{n}, \quad A_{n}=A\left(u_{n}\right), \\
u_{n+1}=\mathrm{e}^{h A_{n 1}} u_{n}, \quad A_{n 1}=A\left(U_{n 1}\right), \quad n \geq 0 .
\end{gathered}
$$

Here, similarly as for Runge-Kutta methods, the numerical solution $u_{n+1}$ is computed by means of an additional internal stage $U_{n 1}$, which is a first-order approximation to the exact solution value at the midpoint $t_{n 1}=t_{n}+h / 2$.

Provided that the exponential is available, the benefits of the Magnus-type integrator (3.1) are its explicitness and favourable stability properties. Namely, the utilisation of exponentials instead of rational functions enhances the stability properties of the integrator. In this respect, we refer to González \& Palencia 13] where the stability and convergence behaviour of Runge-Kutta time discretisations for quasi-linear parabolic problems is studied. However, in [13] the requirement of strong $A(\theta)$-stability implies that the Runge-Kutta method is implicit.

In the nonstiff case, by employing Taylor series expansions, it is straightforward to prove that the numerical method (3.1) has classical order two. In the situation where (1.1) constitutes an abstract quasi-linear parabolic problem on a Banach space, its convergence behaviour is analysed in Section 5

Remark 3.1. We note that the solution of (3.1) remains well defined in $X_{\beta} \cap V$ for any $\gamma<\beta \leq 1$. Namely, whenever $u_{n}$ lies in $X_{\beta} \cap V$ it follows from (2.6) that $U_{n 1}$ is bounded in $X_{\beta}$

$$
\left\|U_{n 1}\right\|_{X_{\beta}} \leq M\left\|u_{n}\right\|_{X_{\beta}} .
$$

On the other hand, for $h>0$ sufficiently small it holds

$$
\left\|U_{n 1}-u_{n}\right\|_{X_{\gamma}} \leq\left\|\mathrm{e}^{h / 2 A_{n}}-I\right\|_{X_{\gamma} \leftarrow X_{\beta}}\left\|u_{n}\right\|_{X_{\beta}} \leq M h^{\beta-\gamma}\left\|u_{n}\right\|_{X_{\beta}} \leq \varrho,
$$

that is, the internal stage $U_{n 1}$ is contained in a ball $B_{\gamma}\left(u_{n}, \varrho\right) \subset X_{\gamma}$ and thus in $V$ for suitably chosen $\varrho>0$; see also (2.7) and (2.9). In particular, it follows $U_{n 1} \in X_{\beta} \cap V$, and therefore the sectorial operator $A\left(U_{n 1}\right)$ is well defined. Now, similar considerations to before show that also $u_{n+1}$ belongs to $X_{\beta} \cap V$.

For a family $\left(F_{i}\right)_{i \geq 0}$ of noncommutative operators on a Banach space, we employ the product notation

$$
\prod_{i=m}^{n} F_{i}=F_{n} F_{n-1} \cdots F_{m}, \quad n \geq m, \quad \prod_{i=m}^{n} F_{i}=I, \quad n<m .
$$

As a consequence, by solving the recursion for the numerical solution in (3.1), we get the relation

$$
u_{n+1}=\prod_{i=0}^{n} \mathrm{e}^{h A_{i 1}} u_{0}=\mathrm{e}^{h A_{n 1}} \mathrm{e}^{h A_{n-1,1}} \cdots \mathrm{e}^{h A_{01}} u_{0}, \quad n \geq 0 .
$$

Our first objective is to study the stability behaviour of this numerical approximation. This is done in Section 4 . 


\section{STABility}

In this section, we analyse the stability behaviour of the numerical method (3.1); that is, we study the dependence of the numerical approximation on the initial value and the effect of additional perturbations. Several auxiliary estimates are collected in Subsection 4.2 .

4.1. Stability result. For the following considerations we employ the assumptions and notation introduced in the previous Sections 2 and 3 . In particular, we denote by $0 \leq \gamma<1$ and $0 \leq \vartheta<1$ the constants specified in Hypothesis 2.1. Further, in view of Example 2.3 and the discussion in Subsection 6.2 it is senseful to suppose $\vartheta \leq \gamma$.

Henceforth, we fix $\gamma<\beta \leq 1$ and $u_{0} \in X_{\beta}$. According to our numerical scheme (3.1) for initial values $v_{0}, w_{0} \in X_{\beta}$ and additional perturbations $p_{n}, q_{n} \in X_{\beta}$ for $n \geq 1$, we consider the recursions

$$
\begin{gathered}
v_{n+1}=\mathrm{e}^{h A\left(V_{n 1}\right)} v_{n}+h p_{n+1}, \quad V_{n 1}=\mathrm{e}^{h / 2 A\left(v_{n}\right)} v_{n}, \\
w_{n+1}=\mathrm{e}^{h A\left(W_{n 1}\right)} w_{n}+h q_{n+1}, \quad W_{n 1}=\mathrm{e}^{h / 2 A\left(w_{n}\right)} w_{n}, \quad n \geq 0 .
\end{gathered}
$$

We note that similar considerations as in Remark 3.1 imply that $V_{n 1}$ and $v_{n+1}$ belong to $X_{\beta} \cap V$ provided that $v_{n} \in X_{\beta} \cap V, p_{n+1} \in X_{\beta}$ is bounded, and $h>0$ is sufficiently small. The analogue is valid for $w_{n+1}$.

Furthermore, the following result shows that these recurrence formulas remain bounded in $X_{\beta}$. Especially, it follows that the Magnus-type integrator (3.1) starting from $u_{0} \in X_{\beta} \cap V$ is applicable up to time $T$.

Theorem 4.1 (Stability). Suppose that Hypothesis 2.1 is fulfilled with $\vartheta>0$. For $\gamma<\beta \leq 1$ let $v_{0} \in X_{\beta} \cap V$ and $w_{0} \in X_{\beta} \cap V$ and assume that $p_{n}$ and $q_{n}$ are bounded in $X_{\beta}$ for $n \geq 1$. Then, for $h>0$ chosen sufficiently small the solutions of (4.1) satisfy the bound

$$
\left\|v_{n}-w_{n}\right\|_{X_{\beta}} \leq C\left(\left\|v_{0}-w_{0}\right\|_{X_{\beta}}+\max _{1 \leq j \leq n}\left\|p_{j}-q_{j}\right\|_{X_{\beta}}\right), \quad 0 \leq n h \leq T,
$$

with constant $C>0$ not depending on $n$ and $h$.

Proof. Our proof is based on a fixed-point iteration based on a global representation of the solutions in (4.1). For this purpose, we introduce several notations.

For the following, we choose $u_{0} \in X_{\beta}$ and fix $\gamma<\zeta<\beta$ and $0<\alpha<\beta-\zeta$. For constants $\varrho>0$ and $\widetilde{L}>0$ we set

$$
\begin{aligned}
\mathcal{Z}=\{ & \left\{z=\left(z_{n}\right)_{0 \leq n h \leq T}: z_{0} \in B_{\beta}\left(u_{0}, \varrho\right) \cap V, z_{n} \in X_{\zeta} \cap V \text { for } n \geq 1\right. \\
& \left.\quad \text { and } n h \leq T,\left\|z_{n}-z_{m}\right\|_{X_{\zeta}} \leq \widetilde{L}\left(t_{n}-t_{m}\right)^{\alpha} \text { for } 0 \leq m h \leq n h \leq T\right\} .
\end{aligned}
$$

In particular, for $z^{*} \in B_{\beta}\left(u_{0}, \varrho\right) \cap V$, we denote $\mathcal{Z}_{z^{*}}=\left\{z \in \mathcal{Z}: z_{0}=z^{*}\right\}$. Note that the sequence spaces $\mathcal{Z}$ and $\mathcal{Z}_{z^{*}}$ are complete metric spaces with the distance induced by the maximum norm

$$
\|z\|_{X_{\zeta}, \infty}=\max _{0 \leq n h \leq T}\left\|z_{n}\right\|_{X_{\zeta}}
$$

see also (2.10). Besides, for some $\sigma>0$, we introduce the set

$$
\mathcal{S}=\left\{s=\left(s_{n}\right)_{h \leq n h \leq T}: s_{n} \in B_{\beta}(0, \sigma) \text { for } n \geq 1 \text { and } n h \leq T\right\} .
$$


For $z \in \mathcal{Z}$, according to relation (4.1), we denote by $Z_{1}(z)=\left(Z_{n 1}(z)\right)_{n \geq 0}$ the sequence defined through

$$
Z_{n 1}(z)=\mathrm{e}^{h / 2 A\left(z_{n}\right)} z_{n}, \quad n \geq 0 .
$$

Moreover, we introduce a family of linear operators $L(z)=\left(L_{m}^{n}(z)\right)_{n \geq m \geq 0}$ depending on the sequence $Z_{1}$ and thus on $z$

$$
L_{m}^{n}(z)=\prod_{i=m}^{n} \mathrm{e}^{h A\left(Z_{i 1}(z)\right)}, \quad 0 \leq m \leq n .
$$

For the following, we fix $z^{*} \in X_{\beta} \cap V$ and $s \in \mathcal{S}$ and let

$$
\begin{gathered}
\mathcal{N}: \mathcal{Z}_{z^{*}} \longrightarrow \mathcal{Z}_{z^{*}}: z \longmapsto \mathcal{N}(z)=N(z, s)=\left(N_{n}(z, s)\right)_{0 \leq n h \leq T} \\
N_{0}(z, s)=z^{*}, \quad N_{n}(z, s)=L_{0}^{n-1}(z) z^{*}+h \sum_{j=0}^{n-1} L_{j+1}^{n-1}(z) s_{j+1}, \quad n \geq 1 .
\end{gathered}
$$

Clearly, a sequence $z \in \mathcal{Z}_{z^{*}}$ that is a fixed point of the nonlinear operator $\mathcal{N}$, i.e., $z$ satisfies the relation $z=\mathcal{N}(z)$, also fulfills the recurrence formula

$$
z_{n+1}=\mathrm{e}^{h A\left(Z_{n 1}\right)} z_{n}+h s_{n+1}, \quad Z_{n 1}=\mathrm{e}^{h / 2 A\left(z_{n}\right)} z_{n}, \quad n \geq 0,
$$

with initial value $z_{0}=z^{*}$.

We next prove the unique solvability of the fixed-point equation $z=\mathcal{N}(z)$ and the continuous dependence of the fixed point on the initial value and additional perturbations. Several auxiliary results needed for the following considerations are derived in the subsequent Subsection 4.2

(i) Let $v, w \in \mathcal{Z}_{z^{*}}$ and $s \in \mathcal{S}$. Estimating the difference $N_{n}(v, s)-N_{n}(w, s)$ with the help of Lemma 4.6 and using that $\left\|s_{j+1}\right\| \leq \sigma$ for $0 \leq j \leq n-1$ gives

$$
\begin{aligned}
& \left\|N_{n}(v, s)-N_{n}(w, s)\right\|_{X_{\zeta}} \leq\left\|L_{0}^{n-1}(v)-L_{0}^{n-1}(w)\right\|_{X_{\zeta} \leftarrow X_{\beta}}\left\|z^{*}\right\|_{X_{\beta}} \\
& \quad+h \sum_{j=0}^{n-1}\left\|L_{j+1}^{n-1}(v)-L_{j+1}^{n-1}(w)\right\|_{X_{\zeta} \leftarrow X_{\beta}}\left\|s_{j+1}\right\|_{X_{\beta}} \\
& \leq C\left(t_{n-1}^{\beta-\zeta}\left\|z^{*}\right\|_{X_{\beta}}+\sigma h \sum_{j=0}^{n-2}\left(t_{n-1}-t_{j+1}\right)^{\beta-\zeta}\right)\|v-w\|_{X_{\zeta}, \infty} \\
& \leq C t_{n}^{\beta-\zeta}\left(\left\|z^{*}\right\|_{X_{\beta}}+\sigma t_{n}\right)\|v-w\|_{X_{\zeta}, \infty} .
\end{aligned}
$$

If $\beta=1$, an additional logarithmic term $(1+|\log h|)$ arises. Thus, for $0<t_{n} \leq T$ and $h>0$ small enough the mapping $\mathcal{N}$ is contractive; that is, the estimate

$$
\|\mathcal{N}(v)-\mathcal{N}(w)\|_{X_{\zeta}, \infty} \leq \kappa\|v-w\|_{X_{\zeta}, \infty}, \quad v, w \in \mathcal{Z}_{z^{*}}
$$

holds with constant $\kappa<1$. 
(ii) For any $z \in \mathcal{Z}_{z^{*}}$ and $s \in \mathcal{S}$ Lemmas 4.4 and 4.5 imply

$$
\begin{aligned}
& \left\|N_{n}(z, s)-N_{m}(z, s)\right\|_{X_{\zeta}} \leq\left\|L_{0}^{n-1}(z)-L_{0}^{m-1}(z)\right\|_{X_{\zeta} \leftarrow X_{\beta}}\left\|z^{*}\right\|_{X_{\beta}} \\
& \quad+h \sum_{j=0}^{m-1}\left\|L_{j+1}^{n-1}(z)-L_{j+1}^{m-1}(z)\right\|_{X_{\zeta} \leftarrow X_{\beta}}\left\|s_{j+1}\right\|_{X_{\beta}} \\
& \quad+h \sum_{j=m}^{n-1}\left\|L_{j+1}^{n-1}(z)\right\|_{X_{\zeta} \leftarrow X_{\beta}}\left\|s_{j+1}\right\|_{X_{\beta}} \\
& \quad \leq \widetilde{C}\left(1+C t_{n-1}^{\alpha}\right)\left(\left\|z^{*}\right\|_{X_{\beta}}\left(t_{n}-t_{m}\right)^{\beta-\zeta}+\sigma t_{m-1}\left(t_{n}-t_{m}\right)^{\beta-\zeta}+\sigma\left(t_{n-1}-t_{m}\right)\right) \\
& \leq \widetilde{C}\left(1+C t_{n}^{\alpha}\right)\left(t_{n}-t_{m}\right)^{\beta-\zeta}
\end{aligned}
$$

with constant $\widetilde{C}>0$ independent of the Hölder constant $\widetilde{L}$. This relation shows that the constant $\widetilde{L}>0$ can be chosen such that sequence $\mathcal{N}(z)$ belongs to $\mathcal{Z}$ for $T>0$ sufficiently small. Again, if $\beta=1$, an additional logarithmis factor appears in the estimate.

As a consequence, $\mathcal{N}$ is a contraction on $\mathcal{Z}_{z^{*}}$. Therefore, an application of the Banach contraction principle shows that $\mathcal{N}$ possesses a unique fixed point $z \in \mathcal{Z}_{z^{*}}$. Consequently, for any $z^{*} \in X_{\beta} \cap V$ and $s \in \mathcal{S}$ the recursion (4.4) is solvable in the sequence space $\mathcal{Z}_{z^{*}}$.

As a further consequence, we obtain the stability estimate of the theorem. Assume that $v, w \in \mathcal{Z}$ and $p, q \in \mathcal{S}$ fulfill the identities

$$
v=N(v, p), \quad w=N(w, q) .
$$

The bound in (i) together with Lemma 4.4 shows

$$
\begin{aligned}
\|v-w\|_{X_{\zeta}, \infty} & =\|N(v, p)-N(w, q)\|_{X_{\zeta}, \infty} \\
& \leq\|N(v, p)-N(w, p)\|_{X_{\zeta}, \infty}+\|N(w, p)-N(w, q)\|_{X_{\zeta}, \infty} \\
& \leq \kappa\|v-w\|_{X_{\zeta}, \infty}+C\left(\left\|v_{0}-w_{0}\right\|_{X_{\beta}}+\|p-q\|_{X_{\beta}, \infty}\right) .
\end{aligned}
$$

Therefore, as $\kappa<1$ we get the relation

$$
\|v-w\|_{X_{\zeta}, \infty} \leq C\left(\left\|v_{0}-w_{0}\right\|_{X_{\beta}}+\|p-q\|_{X_{\beta}, \infty}\right) .
$$

Applying the above arguments together with the previous estimate finally proves the following bound in $X_{\beta}$

$$
\begin{aligned}
\|v-w\|_{X_{\beta}, \infty} & \leq\|N(v, p)-N(w, p)\|_{X_{\beta}, \infty}+\|N(w, p)-N(w, q)\|_{X_{\beta}, \infty} \\
& \leq C\|v-w\|_{X_{\zeta}, \infty}+C\left(\left\|v_{0}-w_{0}\right\|_{X_{\beta}}+\|p-q\|_{X_{\beta}, \infty}\right) \\
& \leq C\left(\left\|v_{0}-w_{0}\right\|_{X_{\beta}}+\|p-q\|_{X_{\beta}, \infty}\right),
\end{aligned}
$$

which is the desired result.

We finally remark that the rather strong restrictions concerning the size of the end time $T>0$ can be weakened by introducing exponential weights in the maximum norm. Alternatively, combining the stability and the convergence result given in Section 5 shows the validity of Theorem 4.1 on the whole interval of existence of the true solution $u:[0, T] \rightarrow X_{\beta}$ of (1.1).

Remark 4.2. The analogue of Theorem 4.1 is valid for any Magnus-type integrator of the form $u_{n+1}=\mathrm{e}^{h U_{n 1}} u_{n}$ provided that the internal stages $U_{n 1}$ satisfy an estimate 
of the form

$$
\left\|U_{n 1}-U_{m 1}\right\|_{X_{\gamma}} \leq C\left(t_{n}-t_{m}\right)^{\alpha}, \quad 0 \leq t_{m} \leq t_{n} \leq T
$$

see also Lemma 4.3

4.2. Auxiliary estimates. In the rest of this paper, we employ the assumptions and abbrevitations introduced in Subsection 4.1. In particular, as in the proof of Theorem 4.1, we choose $\gamma<\zeta<\beta$ and $0<\alpha<\beta-\zeta$. In this subsection, we denote by $\widetilde{C}>0$ a constant that only depends on the constants that appear in Hypothesis 2.1, but not on the Hölder constant $\widetilde{L}$; see (4.2). Especially, the constants $\widetilde{C}>0$ and $C>0$ are independent of $n$ and $h$.

At first, we show that for any sequence $z \in \mathcal{Z}$ the associated sequence $Z_{1}(z)$ reflects the Hölder continuity of $z$; see also (4.2) and (4.3a). For the moment, as we consider a fixed sequence $z \in \mathcal{Z}$, we omit the dependence of $Z_{1}$ on $z$.

Lemma 4.3. Assume that Hypothesis 2.1 holds with $\vartheta \geq 0$. Then, for any $z \in \mathcal{Z}$ the associated sequence $Z_{1}=\left(Z_{n 1}\right)_{n \geq 0}$ defined by (4.3a) satisfies the estimate

$$
\left\|Z_{n 1}-Z_{m 1}\right\|_{X_{\gamma}} \leq C\left(t_{n}-t_{m}\right)^{\alpha}, \quad 0 \leq t_{m} \leq t_{n} \leq T,
$$

with constant $C>0$.

Proof. In order to estimate the difference $Z_{n 1}-Z_{m 1}$, we make use of the identity

$$
Z_{n 1}-Z_{m 1}=\mathrm{e}^{h / 2 A\left(z_{n}\right)}\left(z_{n}-z_{m}\right)+\left(\mathrm{e}^{h / 2 A\left(z_{n}\right)}-\mathrm{e}^{h / 2 A\left(z_{m}\right)}\right) z_{m} .
$$

Due to the fact that $z$ lies in $\mathcal{Z}$, together with (2.6) it follows for $0 \leq t_{m} \leq t_{n} \leq T$

$$
\left\|\mathrm{e}^{h / 2 A\left(z_{n}\right)}\left(z_{n}-z_{m}\right)\right\|_{X_{\gamma}} \leq\left\|\mathrm{e}^{h / 2 A\left(z_{n}\right)}\right\|_{X_{\gamma} \leftarrow X_{\zeta}}\left\|z_{n}-z_{m}\right\|_{X_{\zeta}} \leq C\left(t_{n}-t_{m}\right)^{\alpha} .
$$

On the other hand, let $\Gamma$ be a path that surrounds the spectrum of the sectorial operators $A\left(z_{n}\right)$ and $A\left(z_{m}\right)$. Then, by means of the integral formula of Cauchy, we have the representation

$$
\begin{aligned}
\left(\mathrm{e}^{h / 2 A\left(z_{n}\right)}-\mathrm{e}^{h / 2 A\left(z_{m}\right)}\right) z_{m}=\frac{h}{\pi \mathrm{i}} & \int_{\Gamma} \mathrm{e}^{\lambda}\left(\lambda I-h / 2 A\left(z_{n}\right)\right)^{-1} \\
& \times\left(A\left(z_{n}\right)-A\left(z_{m}\right)\right)\left(\lambda I-h / 2 A\left(z_{m}\right)\right)^{-1} z_{m} \mathrm{~d} \lambda
\end{aligned}
$$

see also (2.5). We estimate this expression with the help of relation (2.3) and the resolvent bound (2.4). Note further that $\left\|z_{n}-z_{m}\right\|_{X_{\gamma}} \leq K\left\|z_{n}-z_{m}\right\|_{X_{\zeta}}$ with some $K>0$. As a consequence, we get the estimate

$$
\begin{gathered}
\left\|\left(\mathrm{e}^{h / 2 A\left(z_{n}\right)}-\mathrm{e}^{h / 2 A\left(z_{m}\right)}\right) z_{m}\right\|_{X_{\gamma}} \leq \frac{h}{\pi} \int_{\Gamma}\left|\mathrm{e}^{\lambda}\right|\left\|\left(\lambda I-h / 2 A\left(z_{n}\right)\right)^{-1}\right\|_{X_{\gamma} \leftarrow X} \\
\times\left\|A\left(z_{n}\right)-A\left(z_{m}\right)\right\|_{X \leftarrow D}\left\|\left(\lambda I-h / 2 A\left(z_{m}\right)\right)^{-1}\right\|_{D \leftarrow X_{\zeta}}\left\|z_{m}\right\|_{X_{\zeta}}|\mathrm{d} \lambda| \\
\leq C\left\|z_{n}-z_{m}\right\|_{X_{\gamma}}\left\|z_{m}\right\|_{X_{\zeta}} \leq C\left(t_{n}-t_{m}\right)^{\alpha} .
\end{gathered}
$$

Altogether, this yields the desired result.

As a direct consequence of (2.6) we obtain the following bound for the analytic semigroup generated by the sectorial operator $A\left(Z_{m 1}\right)$ :

$$
\left\|\mathrm{e}^{\left(t_{n+1}-t_{m}\right) A\left(Z_{m 1}\right)}\right\|_{X_{\nu} \leftarrow X_{\mu}} \leq M\left(t_{n+1}-t_{m}\right)^{-\nu+\mu}, \quad 0 \leq t_{m} \leq t_{n} \leq T,
$$

whenever $0 \leq \mu \leq \nu \leq 1$. Lemma 4.4 below shows that the corresponding estimate remains valid for $L=L(z)$; see (4.3b). 
Lemma 4.4. Suppose that Hypothesis 2.1 holds with $\vartheta>0$. Then, for any $z \in \mathcal{Z}$ the associated linear operator family $L=\left(L_{m}^{n}\right)_{n \geq m \geq 0}$ defined by (4.3b) fulfills

$$
\left\|L_{m}^{n}\right\|_{X_{\nu} \leftarrow X_{\mu}} \leq \widetilde{C}\left(1+C\left(t_{n+1}-t_{m}\right)^{\alpha}\right)\left(t_{n+1}-t_{m}\right)^{-\nu+\mu}, \quad 0 \leq t_{m} \leq t_{n} \leq T,
$$

for all $0 \leq \mu \leq \nu \leq 1$ provided that $\vartheta<\mu+\alpha$.

Proof. Our techniques for proving Lemma 4.4 are close to that applied in [11, 26]. The basic idea is to compare $L_{m}^{n}$ with the frozen operator

$$
\prod_{i=m}^{n} \mathrm{e}^{h A\left(Z_{m 1}\right)}=\mathrm{e}^{\left(t_{n+1}-t_{m}\right) A\left(Z_{m 1}\right)}
$$

where the bound (4.5) is available. Thus, it remains to estimate the difference

$$
\Delta_{m}^{n}=L_{m}^{n}-\mathrm{e}^{\left(t_{n+1}-t_{m}\right) A\left(Z_{m 1}\right)}, \quad 0 \leq m<n .
$$

From a telescopic identity, we obtain the equality

$$
\Delta_{m}^{n}=\sum_{j=m+1}^{n-1} \Delta_{j+1}^{n} \Xi_{j m}+\sum_{j=m+1}^{n} \mathrm{e}^{\left(t_{n+1}-t_{j+1}\right) A\left(Z_{m 1}\right)} \Xi_{j m},
$$

which involves the linear operator

$$
\Xi_{j m}=\left(\mathrm{e}^{h A\left(Z_{j 1}\right)}-\mathrm{e}^{h A\left(Z_{m 1}\right)}\right) \mathrm{e}^{\left(t_{j}-t_{m}\right) A\left(Z_{m 1}\right)}, \quad j>m .
$$

The integral formula of Cauchy yields the following representation, where the path $\Gamma$ is chosen in such a way that it surrounds the spectrum of the sectorial operators $A\left(Z_{j 1}\right)$ and $A\left(Z_{m 1}\right)$ (see also (2.5) and the proof of Lemma 4.3)

$$
\begin{aligned}
\mathrm{e}^{\left(t_{n+1}-t_{j+1}\right) A\left(Z_{m 1}\right)} \Xi_{j m}= & \frac{h}{2 \pi \mathrm{i}} \int_{\Gamma} \mathrm{e}^{\lambda} \mathrm{e}^{\left(t_{n+1}-t_{j+1}\right) A\left(Z_{m 1}\right)}\left(\lambda I-h A\left(Z_{j 1}\right)\right)^{-1} \\
& \times\left(A\left(Z_{j 1}\right)-A\left(Z_{m 1}\right)\right)\left(\lambda I-h A\left(Z_{m 1}\right)\right)^{-1} \mathrm{e}^{\left(t_{j}-t_{m}\right) A\left(Z_{m 1}\right)} \mathrm{d} \lambda .
\end{aligned}
$$

We estimate this expression by applying the resolvent bound (2.4) and further (2.6). Due to relation (2.3) and Lemma 4.3, for $m<j<n$ we finally get

$$
\begin{gathered}
\left\|\mathrm{e}^{\left(t_{n+1}-t_{j+1}\right) A\left(Z_{m 1}\right)} \Xi_{j m}\right\|_{X_{\nu} \leftarrow X_{\mu}} \leq C h\left(t_{n+1}-t_{j+1}\right)^{-\nu+\vartheta}\left(t_{j}-t_{m}\right)^{-1-\vartheta+\mu} \\
\times\left\|Z_{j 1}-Z_{m 1}\right\|_{X_{\gamma}} \\
\leq C h\left(t_{n+1}-t_{j+1}\right)^{-\nu+\vartheta}\left(t_{j}-t_{m}\right)^{-1-\vartheta+\mu+\alpha}, \quad m<j<n .
\end{gathered}
$$

Moreover, it follows that

$$
\left\|\Xi_{n m}\right\|_{X_{\nu} \leftarrow X_{\mu}} \leq C h^{1-\nu+\vartheta}\left(t_{n}-t_{m}\right)^{-1-\vartheta+\mu+\alpha} .
$$

Thus, by interpreting the last sum in (4.6b) as a Riemann sum and estimating it by the associated integral, we have

$$
\sum_{j=m+1}^{n-1}\left\|\mathrm{e}^{\left(t_{n+1}-t_{j+1}\right) A\left(Z_{m 1}\right)} \Xi_{j m}\right\|_{X_{\nu} \leftarrow X_{\mu}} \leq C\left(t_{n+1}-t_{m}\right)^{-\nu+\mu+\alpha},
$$

provided that $\vartheta<\mu+\alpha$. Furthermore, we make use of the relation

$$
\left\|\Xi_{j m}\right\|_{X_{\mu} \leftarrow X_{\mu}} \leq C h^{1-\mu+\vartheta}\left(t_{j}-t_{m}\right)^{-1-\vartheta+\mu+\alpha}, \quad j>m .
$$


First, we estimate $\Delta_{m}^{n}$ as operator from $X_{\vartheta}$ to $X_{\nu}$. With the help of the above relations, due to the fact that for $j>m$ and $n>m$ it holds that

$$
\begin{gathered}
\left\|\Xi_{j m}\right\|_{X_{\vartheta} \leftarrow X_{\vartheta}} \leq C h\left(t_{j}-t_{m}\right)^{-1+\alpha}, \quad\left\|\Xi_{n m}\right\|_{X_{\nu} \leftarrow X_{\vartheta}} \leq C h^{1-\nu+\vartheta}\left(t_{n}-t_{m}\right)^{-1+\alpha}, \\
\sum_{j=m+1}^{n-1}\left\|\mathrm{e}^{\left(t_{n+1}-t_{j+1}\right) A\left(Z_{m 1}\right)} \Xi_{j m}\right\|_{X_{\nu} \leftarrow X_{\vartheta}} \leq C\left(t_{n+1}-t_{m}\right)^{-\nu+\vartheta+\alpha},
\end{gathered}
$$

then we obtain the following bound:

$$
\begin{aligned}
\left\|\Delta_{m}^{n}\right\|_{X_{\nu} \leftarrow X_{\vartheta}} \leq & \sum_{j=m+1}^{n-1}\left\|\Delta_{j+1}^{n}\right\|_{X_{\nu} \leftarrow X_{\vartheta}}\left\|\Xi_{j m}\right\|_{X_{\vartheta} \leftarrow X_{\vartheta}}+\left\|\Xi_{n m}\right\|_{X_{\nu} \leftarrow X_{\vartheta}} \\
& +\sum_{j=m+1}^{n-1}\left\|\mathrm{e}^{\left(t_{n+1}-t_{j+1}\right) A\left(Z_{m 1}\right)} \Xi_{j m}\right\|_{X_{\nu} \leftarrow X_{\vartheta}} \\
\leq & C h \sum_{j=m+1}^{n-1}\left\|\Delta_{j+1}^{n}\right\|_{X_{\nu} \leftarrow X_{\vartheta}}\left(t_{j}-t_{m}\right)^{-1+\alpha}+C\left(t_{n+1}-t_{m}\right)^{-\nu+\vartheta+\alpha} .
\end{aligned}
$$

Thus, from a Gronwall-type inequality with a weakly singular kernel (see, e.g., [8, 24]), it follows that

$$
\left\|\Delta_{m}^{n}\right\|_{X_{\nu} \leftarrow X_{\vartheta}} \leq C h\left(t_{n+1}-t_{m}\right)^{-\nu+\vartheta+\alpha}
$$

with constant $C>0$ possibly depending on $T$. Now, it is straightforward to estimate $\Delta_{m}^{n}$ as operator from $X_{\mu}$ to $X_{\nu}$

$$
\begin{aligned}
\left\|\Delta_{m}^{n}\right\|_{X_{\nu} \leftarrow X_{\mu}} \leq \sum_{j=m+1}^{n-1}\left\|\Delta_{j+1}^{n}\right\|_{X_{\nu} \leftarrow X_{\vartheta}}\left\|\Xi_{j m}\right\|_{X_{\vartheta} \leftarrow X_{\mu}}+\left\|\Xi_{n m}\right\|_{X_{\nu} \leftarrow X_{\mu}} \\
\quad+\sum_{j=m+1}^{n-1}\left\|\mathrm{e}^{\left(t_{n+1}-t_{j+1}\right) A\left(Z_{m 1}\right)} \Xi_{j m}\right\|_{X_{\nu} \leftarrow X_{\mu}} \\
\leq C h \sum_{j=m+1}^{n-1}\left(t_{n+1}-t_{j}\right)^{-\nu+\vartheta+\alpha}\left(t_{j}-t_{m}\right)^{-1-\vartheta+\mu+\alpha}+C\left(t_{n+1}-t_{m}\right)^{-\nu+\mu+\alpha}
\end{aligned}
$$

wherefore we finally have

$$
\left\|\Delta_{m}^{n}\right\|_{X_{\nu} \leftarrow X_{\mu}} \leq C\left(t_{n+1}-t_{m}\right)^{-\nu+\mu+\alpha} .
$$

Together with (4.5) this yields the desired result.

Now, with the help of Lemma 4.4 we are in the position to show that $L$ is Hölder continuous.

Lemma 4.5. In the situation of Lemma 4.4, for $z \in \mathcal{Z}$ the associated operator family $L=\left(L_{m}^{n}\right)_{n \geq m \geq 0}$ satisfies the estimates

$$
\begin{aligned}
& \left\|L_{j}^{n}(z)-L_{j}^{m}(z)\right\|_{X_{\nu} \leftarrow X_{\mu}} \leq \widetilde{C}\left(1+C t_{n}^{\alpha}\right)\left(t_{n}-t_{m}\right)^{-\nu+\mu}, \quad \nu \neq 1, \\
& \left\|L_{j}^{n}(z)-L_{j}^{m}(z)\right\|_{D \leftarrow X_{\mu}} \leq \widetilde{C}\left(1+C t_{n}^{\alpha}\right)(1+|\log h|)\left(t_{n}-t_{m}\right)^{-1+\mu},
\end{aligned}
$$

where $0 \leq \mu, \nu \leq 1$ and $0 \leq t_{j} \leq t_{m}<t_{n} \leq T$. 
Proof. From a telescopic identity, for $j \leq m<n$, we obtain

$$
L_{j}^{n}-L_{j}^{m}=\left(L_{m+1}^{n}-I\right) L_{j}^{m}=\sum_{i=m+1}^{n} L_{i+1}^{n}\left(\mathrm{e}^{h A\left(Z_{i 1}\right)}-I\right) L_{j}^{m}, \quad j \leq m<n .
$$

We note that the relations in (2.8) imply

$$
\left\|\mathrm{e}^{h A\left(Z_{i 1}\right)}-I\right\|_{X \leftarrow D}=\left\|h A\left(Z_{i 1}\right) \varphi\left(h A\left(Z_{i 1}\right)\right)\right\|_{X \leftarrow D} \leq M h, \quad 0 \leq t_{i} \leq T,
$$

see also [25], for example. Further, it holds

$$
\left\|\mathrm{e}^{h A\left(Z_{i 1}\right)}-I\right\|_{X_{\nu} \leftarrow D} \leq M h^{1-\nu}, \quad 0 \leq t_{i} \leq T .
$$

Thus, by applying the bound from Lemma 4.4, for any $0 \leq \mu, \nu \leq 1$, we get

$$
\begin{aligned}
\left\|L_{j}^{n}-L_{j}^{m}\right\|_{X_{\nu} \leftarrow X_{\mu}} \leq & \sum_{i=m+1}^{n-1}\left\|L_{i+1}^{n}\right\|_{X_{\nu} \leftarrow X}\left\|\mathrm{e}^{h A\left(Z_{i 1}\right)}-I\right\|_{X \leftarrow D}\left\|L_{j}^{m}\right\|_{D \leftarrow X_{\mu}} \\
& \quad\left\|\mathrm{e}^{h A\left(Z_{i 1}\right)}-I\right\|_{X_{\nu} \leftarrow D}\left\|L_{j}^{m}\right\|_{D \leftarrow X_{\mu}} \\
\leq & \widetilde{C}\left(1+C t_{n}^{\alpha}\right) h \sum_{i=m+1}^{n-1}\left(t_{n+1}-t_{i+1}\right)^{-\nu}\left(t_{m+1}-t_{j}\right)^{-1+\mu} .
\end{aligned}
$$

Therefore, interpreting the sum as a Riemann sum and estimating it by the associated integral yields for $\nu \neq 1$

$$
\begin{aligned}
\left\|L_{j}^{n}-L_{j}^{m}\right\|_{X_{\nu} \leftarrow X_{\mu}} & \leq \widetilde{C}\left(1+C t_{n}^{\alpha}\right)\left(t_{n+1}-t_{m+1}\right)^{1-\nu}\left(t_{m+1}-t_{j}\right)^{-1+\mu} \\
& \leq \widetilde{C}\left(1+C t_{n}^{\alpha}\right)\left(t_{n}-t_{m}\right)^{-\nu+\mu}\left(\frac{n-m}{m+1-j}\right)^{1-\mu} \\
& \leq \widetilde{C}\left(1+C t_{n}^{\alpha}\right)\left(t_{n}-t_{m}\right)^{-\nu+\mu}
\end{aligned}
$$

which proves the desired result. If $\nu=1$, the additional term $(1+|\log h|)$ arises in the estimate.

In Lemma 4.6 we study the dependence of the operators $L_{m}^{n}(z)$ on $z$. For that purpose, for $v=\left(v_{n}\right)_{n \geq 0}$ and $w=\left(w_{n}\right)_{n \geq 0}$ in $\mathcal{Z}$ we denote by $\|v-w\|_{X_{\zeta}, \infty}$ the maximum value of $\left\|v_{n}-w_{n}\right\|_{X_{\zeta}}$ for $0 \leq n h \leq T$; see also (2.10).

Lemma 4.6. Suppose that Hypothesis 2.1 is satisfied with $\vartheta>0$. Then, for sequences $v=\left(v_{n}\right)_{n \geq 0} \in \mathcal{Z}$ and $w=\left(w_{n}\right)_{n \geq 0} \in \mathcal{Z}$ the following estimates are valid for arbitrary $0 \leq \mu \leq \nu \leq 1$ and $0 \leq t_{m}<t_{n} \leq T$. If $\nu \neq 1$ and $\mu \neq 0$, it follows that

$$
\left\|L_{m}^{n}(v)-L_{m}^{n}(w)\right\|_{X_{\nu} \leftarrow X_{\mu}} \leq C\left(t_{n}-t_{m}\right)^{-\nu+\mu}\|v-w\|_{X_{\zeta}, \infty},
$$

else if $\nu=1$ or $\mu=0$, the bound

$$
\left\|L_{m}^{n}(v)-L_{m}^{n}(w)\right\|_{X_{\nu} \leftarrow X_{\mu}} \leq C\left(t_{n}-t_{m}\right)^{-\nu+\mu}(1+|\log h|)\|v-w\|_{X_{\zeta}, \infty}
$$

holds.

Proof. For $v=\left(v_{n}\right)_{n \geq 0} \in \mathcal{Z}$ and $w=\left(w_{n}\right)_{n \geq 0} \in \mathcal{Z}$ we define the associated sequences $V_{1}=\left(V_{n 1}\right)_{n \geq 0}$ and $W_{1}=\left(W_{n 1}\right)_{n \geq 0}$ according to 4.3a). An application of the telescopic identity yields

$$
L_{m}^{n}(v)-L_{m}^{n}(w)=\sum_{j=m}^{n} L_{j+1}^{n}(v)\left(\mathrm{e}^{h A\left(V_{j 1}\right)}-\mathrm{e}^{h A\left(W_{j 1}\right)}\right) L_{m}^{j-1}(w) ;
$$


see also the proof of the previous Lemma 4.5. We estimate $L_{m}^{n}(v)-L_{m}^{n}(w)$ as operator from $X_{\mu}$ to $X_{\nu}$

$$
\begin{gathered}
\left\|L_{m}^{n}(v)-L_{m}^{n}(w)\right\|_{X_{\nu} \leftarrow X_{\mu}} \leq\left\|L_{m+1}^{n}(v)\right\|_{X_{\nu} \leftarrow X}\left\|\mathrm{e}^{h A\left(V_{m 1}\right)}-\mathrm{e}^{h A\left(W_{m 1}\right)}\right\|_{X \leftarrow X_{\mu}} \\
+\sum_{j=m+1}^{n-1}\left\|L_{j+1}^{n}(v)\right\|_{X_{\nu} \leftarrow X}\left\|\mathrm{e}^{h A\left(V_{j 1}\right)}-\mathrm{e}^{h A\left(W_{j 1}\right)}\right\|_{X \leftarrow D}\left\|L_{m}^{j-1}(w)\right\|_{D \leftarrow X_{\mu}} \\
\quad+\left\|\mathrm{e}^{h A\left(V_{n 1}\right)}-\mathrm{e}^{h A\left(W_{n 1}\right)}\right\|_{X_{\nu} \leftarrow D}\left\|L_{m}^{n-1}(w)\right\|_{D \leftarrow X_{\mu}} \cdot
\end{gathered}
$$

By the integral formula of Cauchy, we have the representation

$$
\begin{aligned}
\mathrm{e}^{h A\left(V_{j 1}\right)}-\mathrm{e}^{h A\left(W_{j 1}\right)}=\frac{h}{\pi \mathrm{i}} & \int_{\Gamma} \mathrm{e}^{\lambda}\left(\lambda I-h A\left(V_{j 1}\right)\right)^{-1} \\
& \times\left(A\left(V_{j 1}\right)-A\left(W_{j 1}\right)\right)\left(\lambda I-h A\left(W_{j 1}\right)\right)^{-1} \mathrm{~d} \lambda ;
\end{aligned}
$$

see also (2.5) . Consequently, with the help of (2.4) and (2.6) we have

$$
\begin{gathered}
\left\|\mathrm{e}^{h A\left(V_{j 1}\right)}-\mathrm{e}^{h A\left(W_{j 1}\right)}\right\|_{X \leftarrow X_{\mu}} \leq C h^{\mu}\left\|A\left(V_{j 1}\right)-A\left(W_{j 1}\right)\right\|_{X \leftarrow D}, \quad 0 \leq \mu \leq 1, \\
\left\|\mathrm{e}^{h A\left(V_{j 1}\right)}-\mathrm{e}^{h A\left(W_{j 1}\right)}\right\|_{X_{\nu} \leftarrow D} \leq C h^{1-\nu}\left\|A\left(V_{j 1}\right)-A\left(W_{j 1}\right)\right\|_{X \leftarrow D}, \quad 0 \leq \nu \leq 1 .
\end{gathered}
$$

Hypothesis 2.1 and similar considerations as in the proof of Lemma 4.3 yield the bound

$$
\begin{aligned}
\left\|A\left(V_{n 1}\right)-A\left(W_{n 1}\right)\right\|_{X \leftarrow D} & \leq L\left\|V_{n 1}-W_{n 1}\right\|_{X_{\gamma}} \\
& =L\left\|\mathrm{e}^{h / 2 A\left(v_{n}\right)} v_{n}-\mathrm{e}^{h / 2 A\left(w_{n}\right)} w_{n}\right\|_{X_{\gamma}} \leq C\left\|v_{n}-w_{n}\right\|_{X_{\zeta}} .
\end{aligned}
$$

As a consequence, by means of Lemma 4.4 we finally have

$$
\left\|L_{m}^{n}(v)-L_{m}^{n}(w)\right\|_{X_{\nu} \leftarrow X_{\mu}} \leq C\left(t_{n}-t_{m}\right)^{-\nu+\mu}\|v-w\|_{X_{\zeta}, \infty} .
$$

Here, an additional logarithmic factor arises if $\nu=1$ or $\mu=0$. This proves the desired result.

\section{Convergence}

In this section, we state a convergence result for the Magnus-type integrator (3.1) applied to the quasi-linear problem (1.1). Our proof relies on a favourable relation for the global error that we derive first.

5.1. Relation for error. For subsequent consideration, we employ the abbreviations introduced before in Sections 2 and 3 In particular, for a constant stepsize $h>0$, we let $t_{n}=n h$ and $t_{n 1}=t_{n}+h / 2$, and we set $A_{n}=A\left(u_{n}\right)$ and $A_{n 1}=A\left(U_{n 1}\right)$ for $n \geq 0$. Furthermore, we define

$$
\varphi_{n 1}=\varphi\left(h A_{n 1}\right), \quad \psi_{n 1}=\psi\left(h A_{n 1}\right), \quad \chi_{n 1}=\chi\left(h A_{n 1}\right), \quad \psi_{n}=\left(h / 2 A_{n}\right) ;
$$

see also (2.8a). Besides, it is convenient to denote the exact solution values by

$$
\widehat{u}_{n+1}=u\left(t_{n+1}\right), \quad \widehat{U}_{n 1}=u\left(t_{n 1}\right), \quad \widehat{A}_{n}=A\left(\widehat{u}_{n}\right), \quad \widehat{A}_{n 1}=A\left(\widehat{U}_{n 1}\right) .
$$

Then, the global error of the numerical approximation and the internal stage, respectively, equals

$$
e_{n+1}=u_{n+1}-\widehat{u}_{n+1}, \quad E_{n 1}=U_{n 1}-\widehat{U}_{n 1}, \quad n \geq 0 .
$$


Moreover, the discrete evolution operator is given by

$$
\mathcal{E}_{m}^{n}=\prod_{i=m}^{n} \mathrm{e}^{h A_{i 1}}, \quad 0 \leq m \leq n .
$$

In addition, we set $\mathcal{E}_{m}^{n}=I$ if $n<m$.

In order to represent the global error $e_{n+1}$ in a suitable way, we consider the differential equation (1.1) on the subinterval $\left[t_{n}, t_{n+1}\right]$, and we rewrite the righthand side by adding and substracting $A_{n 1}$

$$
u^{\prime}(t)=A_{n 1} u(t)+g_{n}(t), \quad g_{n}(t)=\left(A(u(t))-A_{n 1}\right) u(t) .
$$

Thus, with the help of the variation-of-constants formula, a relation similar to the second formula in (3.1), involving further the defect of the method, follows:

$$
\widehat{u}_{n+1}=\mathrm{e}^{h A_{n 1}} \widehat{u}_{n}+d_{n+1}, \quad d_{n+1}=\int_{0}^{h} \mathrm{e}^{(h-\tau) A_{n 1}} g_{n}\left(t_{n}+\tau\right) \mathrm{d} \tau .
$$

By taking the difference of (3.1) and (5.2) and resolving the resulting recursion for $e_{n+1}$, we finally obtain

$$
e_{n+1}=\mathcal{E}_{0}^{n} e_{0}-\sum_{j=0}^{n} \mathcal{E}_{j+1}^{n} d_{j+1} .
$$

For deriving a useful relation for the defects, we decompose $g_{n}$ as

$$
g_{n}(t)=f_{n}(t)+\left(\widehat{A}_{n 1}-A_{n 1}\right) u(t), \quad f_{n}(t)=\left(A(u(t))-\widehat{A}_{n 1}\right) u(t) .
$$

Provided that the map $A$ and the exact solution $u$ satisfy suitable regularity assumptions, a Taylor series expansion of $f_{n}:\left[t_{n}, t_{n+1}\right] \rightarrow X$ yields

$$
f_{n}\left(t_{n}+\tau\right)=(\tau-h / 2) f_{n}^{\prime}\left(t_{n 1}\right)+(\tau-h / 2)^{2} \int_{0}^{1}(1-\sigma) f_{n}^{\prime \prime}\left(t_{n 1}+\sigma(\tau-h / 2)\right) \mathrm{d} \sigma,
$$

and, moreover, the identity

$$
A_{n 1}-\widehat{A}_{n 1}=\mathcal{A}_{n 1} E_{n 1}, \quad \mathcal{A}_{n 1}=\int_{0}^{1} A^{\prime}\left(\sigma U_{n 1}+(1-\sigma) \widehat{U}_{n 1}\right) \mathrm{d} \sigma
$$

is valid with $A^{\prime}(v): V \rightarrow L(D, X)$ denoting the Fréchet derivative of $A$ at $v \in V$. Consequently, by integrating according to (5.2) and applying (2.8a), the defects split up into $d_{n+1}=\delta_{n+1}+\theta_{n+1}=\delta_{n+1}^{(0)}+\delta_{n+1}^{(1)}+\theta_{n+1}$ where

$$
\begin{gathered}
\delta_{n+1}^{(0)}=h^{2}\left(\psi_{n 1}-1 / 2 \varphi_{n 1}\right) f_{n}^{\prime}\left(t_{n 1}\right)=h^{3} A_{n 1} \chi_{n 1} f_{n}^{\prime}\left(t_{n 1}\right), \\
\delta_{n+1}^{(1)}=\int_{0}^{h} \mathrm{e}^{(h-\tau) A_{n 1}}(\tau-h / 2)^{2} \int_{0}^{1}(1-\sigma) f_{n}^{\prime \prime}\left(t_{n 1}+\sigma(\tau-h / 2)\right) \mathrm{d} \sigma \mathrm{d} \tau, \\
\theta_{n+1}=-\int_{0}^{h} \mathrm{e}^{(h-\tau) A_{n 1}} \mathcal{A}_{n 1} E_{n 1} u\left(t_{n}+\tau\right) \mathrm{d} \tau .
\end{gathered}
$$

As the term $\theta_{n+1}$ involves the error of the internal stage, we next derive a suitable relation for $E_{n 1}$. Rewriting again the right-hand side of (1.1)

$$
u^{\prime}(t)=A_{n} u(t)+G_{n}(t), \quad G_{n}(t)=\left(A(u(t))-A_{n}\right) u(t),
$$

by the variation-of-constants formula, we obtain the representation

$$
\widehat{U}_{n 1}=\mathrm{e}^{h / 2 A_{n}} \widehat{u}_{n}+D_{n 1}, \quad D_{n 1}=\int_{0}^{h / 2} \mathrm{e}^{(h / 2-\tau) A_{n}} G_{n}\left(t_{n}+\tau\right) \mathrm{d} \tau,
$$


and, together with the first formula in (3.1) this implies

$$
E_{n 1}=\mathrm{e}^{h / 2 A_{n}} e_{n}-D_{n 1} .
$$

Similarly to before, we employ a decomposition of $G_{n}$

$$
G_{n}(t)=F_{n}(t)+\left(\widehat{A}_{n}-A_{n}\right) u(t), \quad F_{n}(t)=\left(A(u(t))-\widehat{A}_{n}\right) u(t),
$$

and thus obtain from a Taylor series expansion the identity

$$
F_{n}\left(t_{n}+\tau\right)=\tau F_{n}^{\prime}\left(t_{n}\right)+\tau^{2} \int_{0}^{1}(1-\sigma) F_{n}^{\prime \prime}\left(t_{n}+\sigma \tau\right) \mathrm{d} \sigma,
$$

and, on the other hand, the relation

$$
A_{n}-\widehat{A}_{n}=\mathcal{A}_{n} e_{n}, \quad \mathcal{A}_{n}=\int_{0}^{1} A^{\prime}\left(\sigma u_{n}+(1-\sigma) \widehat{u}_{n}\right) \mathrm{d} \sigma,
$$

follows. Consequently, determining the integral in (5.6) with the help of (2.8a), yields the splitting $D_{n 1}=\Delta_{n 1}+\Theta_{n 1}=\Delta_{n 1}^{(0)}+\Delta_{n 1}^{(1)}+\Theta_{n 1}$ for the defect of the internal stage where

$$
\begin{gathered}
\Delta_{n 1}^{(0)}=h^{2} / 4 \psi_{n} F_{n}^{\prime}\left(t_{n}\right), \\
\Delta_{n 1}^{(1)}=\int_{0}^{h / 2} \mathrm{e}^{(h / 2-\widetilde{\tau}) A_{n}} \widetilde{\tau}^{2} \int_{0}^{1}(1-\sigma) F_{n}^{\prime \prime}\left(t_{n}+\sigma \widetilde{\tau}\right) \mathrm{d} \sigma \mathrm{d} \widetilde{\tau}, \\
\Theta_{n 1}=-\int_{0}^{h / 2} \mathrm{e}^{(h / 2-\widetilde{\tau}) A_{n}} \mathcal{A}_{n} e_{n} u\left(t_{n}+\widetilde{\tau}\right) \mathrm{d} \widetilde{\tau} .
\end{gathered}
$$

Finally, we expand relation (5.3) by successively inserting formula (5.5c) for the defect $d_{n+1}=\delta_{n+1}+\theta_{n+1}$, formula (5.7), and further (5.9c) for $D_{n 1}=\Delta_{n 1}+\Theta_{n 1}$. Altogether, we have the following representation for the global error

$$
\begin{aligned}
e_{n}= & \mathcal{E}_{0}^{n-1} e_{0}+\sum_{j=0}^{n-1} \mathcal{E}_{j+1}^{n-1} \int_{0}^{h} \mathrm{e}^{(h-\tau) A_{j 1}} \mathcal{A}_{j 1} \\
& \times \int_{0}^{h / 2} \mathrm{e}^{(h / 2-\widetilde{\tau}) A_{j}} \mathcal{A}_{j} e_{j} u\left(t_{j}+\widetilde{\tau}\right) \mathrm{d} \widetilde{\tau} u\left(t_{j}+\widetilde{\tau}\right) \mathrm{d} \widetilde{\tau} u\left(t_{j}+\tau\right) \mathrm{d} \tau \\
& +\sum_{j=0}^{n-1} \mathcal{E}_{j+1}^{n-1} \int_{0}^{h} \mathrm{e}^{(h-\tau) A_{j 1}} \mathcal{A}_{j 1} \mathrm{e}^{h / 2} A_{j} e_{j} u\left(t_{j}+\tau\right) \mathrm{d} \tau \\
& -\sum_{j=0}^{n-1} \mathcal{E}_{j+1}^{n-1} \delta_{j+1}-\sum_{j=0}^{n-1} \mathcal{E}_{j+1}^{n-1} \int_{0}^{h} \mathrm{e}^{(h-\tau) A_{j 1}} \mathcal{A}_{j 1} \Delta_{j 1} u\left(t_{j}+\tau\right) \mathrm{d} \tau,
\end{aligned}
$$

where the defects $\delta_{j+1}=\delta_{j+1}^{(0)}+\delta_{j+1}^{(1)}$ and $\Delta_{j 1}=\Delta_{j 1}^{(0)}+\Delta_{j 1}^{(1)}$ are defined through the formulas (5.5a) $-(5.5 \mathrm{~b})$ and (5.9a) $-(5.9 \mathrm{~b})$.

5.2. Error estimate. We next analyse the error behaviour of the Magnus-type integrator (3.1) for the quasi-linear parabolic problem (1.1) and state a convergence estimate with respect to the norm of the intermediate space $X_{\beta}$ where $\gamma<\beta<1$.

For the derivation of Theorem 5.1, our main tools are the global representation (5.10) as well as the stability estimate of Theorem 4.1. In order to obtain the optimal convergence order, we further make use of a refined stability bound specified in Lemma 5.2 at the end of this subsection. Regarding the error estimate it is notable that the differentiability of the functions $f_{n}$ and $F_{n}$ introduced 
in (5.4) and (5.8) is governed by the smoothness of the exact solution $u$ and the operator family $A$ (that is, the requirement that the first derivatives of $f_{n}$ and $F_{n}$ are bounded in $X_{\vartheta}$ for a certain $\vartheta>0$ ) is satisfied in various applications; see also Subsection 6.2. We finally note that the restriction $\beta<1$ makes sense in view of Remark 3.1] however, the statement of Theorem 5.1 remains valid for the limiting case $\beta=1$.

In the rest of this paper, for maps $g:[0, T] \rightarrow X$ and $G_{j}:\left[t_{j}, t_{j+1}\right] \rightarrow X$ defined for integers $j \geq 0$, we employ the abbreviations

$$
\|g\|_{X, \infty}=\max _{0 \leq t \leq t_{n}}\|g(t)\|_{X}, \quad\|G\|_{X, \infty}=\max _{0 \leq j \leq n-1}\left\|G_{j}\right\|_{X, \infty}
$$

where $\left\|G_{j}\right\|_{X, \infty}=\max \left\{\left\|G_{j}(t)\right\|_{X}: t_{j} \leq t \leq t_{j+1}\right\} ;$ see also (2.10).

Theorem 5.1 (Convergence). Suppose that Hypothesis 2.1 is fulfilled for constants $0<\vartheta \leq \gamma<1$ and choose $\gamma<\beta<1$. Assume further that the exact solution of (1.1) is bounded in $X_{1+\vartheta}$ and that $A^{\prime}(v): V \rightarrow L\left(X_{1+\vartheta}, X_{\vartheta}\right)$ is bounded for every $v \in V$. Besides, we require $u:[0, T] \rightarrow X_{\beta}$ to be Lipschitz continuous with respect to $t$. Then, for $h>0$ chosen sufficiently small, the numerical method (3.1) applied to the abstract initial value problem (1.1) satisfies the convergence estimate

$$
\begin{aligned}
\left\|u_{n}-u\left(t_{n}\right)\right\|_{X_{\beta} \leq} & C\left\|u_{0}-u(0)\right\|_{X_{\beta}}, \\
& +C h^{2-\beta+\vartheta}\left((1+|\log h|)\left\|f^{\prime}\right\|_{X_{\vartheta}, \infty}+\left\|F^{\prime}\right\|_{X_{\vartheta}, \infty}\right) \\
& +C h^{2}\left(\left\|f^{\prime \prime}\right\|_{X, \infty}+h^{1-\beta}\left\|F^{\prime \prime}\right\|_{X, \infty}\right), \quad 0 \leq t_{n} \leq T
\end{aligned}
$$

provided that the quantities on the right-hand side are well defined. The constant $C>0$ is independent of $n$ and $h$.

Proof. We note that the existence of the numerical solution in $X_{\beta}$ is ensured by Theorem 4.1. Thus, it remains to derive the desired convergence bound. For this purpose, we consider relation (5.10) for the global error $e_{n}$ and estimate it in $X_{\beta}$. On the one hand, for the error terms involving the initial values and $e_{j}, 0 \leq j \leq n-1$, we thus obtain the bound

$$
\begin{aligned}
&\left\|e_{n}^{(0)}\right\|_{X_{\beta}} \leq\left\|\mathcal{E}_{0}^{n-1}\right\|_{X_{\beta} \leftarrow X_{\beta}}\left\|e_{0}\right\|_{X_{\beta}} \\
&++\sum_{j=0}^{n-1} \int_{0}^{h} \int_{0}^{h / 2}\left\|\mathcal{E}_{j+1}^{n-1} \mathrm{e}^{(h-\tau) A_{j 1}}\right\|_{X_{\beta} \leftarrow X_{\vartheta}}\left\|\mathcal{A}_{j 1}\right\|_{L\left(X_{1+\vartheta}, X_{\vartheta}\right) \leftarrow X_{\gamma}} \\
& \times\left\|\mathrm{e}^{(h / 2-\widetilde{\tau}) A_{j}}\right\|_{X_{\gamma} \leftarrow X_{\vartheta}}\left\|\mathcal{A}_{j}\right\|_{L\left(X_{1+\vartheta}, X_{\vartheta}\right) \leftarrow X_{\gamma}}\left\|e_{j}\right\|_{X_{\beta}} \\
& \times\left\|u\left(t_{j}+\widetilde{\tau}\right)\right\|_{X_{1+\vartheta}}\left\|u\left(t_{j}+\tau\right)\right\|_{X_{1+\vartheta}} \mathrm{d} \widetilde{\tau} \mathrm{d} \tau \\
&+\sum_{j=0}^{n-1} \int_{0}^{h}\left\|\mathcal{E}_{j+1}^{n-1} \mathrm{e}^{(h-\tau) A_{j 1}}\right\|_{X_{\beta} \leftarrow X_{\vartheta}}\left\|\mathcal{A}_{j 1}\right\|_{L\left(X_{1+\vartheta}, X_{\vartheta}\right) \leftarrow X_{\gamma}} \\
& \quad \times\left\|\mathrm{e}^{h / 2 A_{j}}\right\|_{X_{\gamma} \leftarrow X_{\beta}}\left\|e_{j}\right\|_{X_{\beta}}\left\|u\left(t_{j}+\tau\right)\right\|_{X_{1+\vartheta}} \mathrm{d} \tau .
\end{aligned}
$$


On the other hand, inserting the representation (5.5a) for the defects $\delta_{n+1}^{(0)}$ yields the following estimate for the remaining terms

$$
\begin{aligned}
\left\|e_{n}^{(1)}\right\|_{X_{\beta}} \leq & h^{3} \sum_{j=0}^{n-2}\left\|\mathcal{E}_{j+1}^{n-1} A_{j 1} \chi_{j 1}\right\|_{X_{\beta} \leftarrow X_{\vartheta}}\left\|f_{j}^{\prime}\left(t_{j 1}\right)\right\|_{X_{\vartheta}} \\
& +h^{2}\left(\left\|\psi_{n-1,1}\right\|_{X_{\beta} \leftarrow X_{\vartheta}}+1 / 2\left\|\varphi_{n-1,1}\right\|_{X_{\beta} \leftarrow X_{\vartheta}}\right)\left\|f_{n-1}^{\prime}\left(t_{n-1,1}\right)\right\|_{X_{\vartheta}} \\
& +\sum_{j=0}^{n-2}\left\|\mathcal{E}_{j+1}^{n-1}\right\|_{X_{\beta} \leftarrow X}\left\|\delta_{j+1}^{(1)}\right\|_{X}+\left\|\delta_{n}^{(1)}\right\|_{X_{\beta}} \\
& +\sum_{j=0}^{n-1} \int_{0}^{h}\left\|\mathcal{E}_{j+1}^{n-1} \mathrm{e}^{(h-\tau) A_{j 1}}\right\|_{X_{\beta} \leftarrow X_{\vartheta}} \\
& \times\left\|\mathcal{A}_{j 1}\right\|_{L\left(X_{1+\vartheta}, X_{\vartheta}\right) \leftarrow X_{\gamma}}\left\|\Delta_{j 1}\right\|_{X_{\beta}}\left\|u\left(t_{j}+\tau\right)\right\|_{X_{1+\vartheta}} \mathrm{d} \tau .
\end{aligned}
$$

We next apply the bounds for the analytic semigroup and the related operators (see (2.6) and (2.8b) ), as well as the stability bounds of Lemma 5.2, Note further that for any $0 \leq \mu \leq 1$ the relation

$$
\begin{aligned}
\left\|\delta_{n+1}^{(1)}\right\|_{X_{\mu}} \leq & \int_{0}^{h} \int_{0}^{1}|\tau-h / 2|^{2}\left\|\mathrm{e}^{(h-\tau) A_{n 1}}\right\|_{X_{\mu} \leftarrow X} \\
& \times\left\|f_{n}^{\prime \prime}\left(t_{n 1}+\sigma(\tau-h / 2)\right)\right\|_{X} \mathrm{~d} \sigma \mathrm{d} \tau \leq C h^{3-\mu}\left\|f^{\prime \prime}\right\|_{X, \infty}
\end{aligned}
$$

holds, and moreover that we have

$$
\begin{aligned}
&\left\|\Delta_{n 1}\right\|_{X_{\beta}} \leq h^{2}\left\|\psi_{n}\right\|_{X_{\beta} \leftarrow X_{\vartheta}}\left\|F_{n}^{\prime}\left(t_{n}\right)\right\|_{X_{\vartheta}} \\
& \quad+\int_{0}^{h / 2} \int_{0}^{1} \widetilde{\tau}^{2}\left\|\mathrm{e}^{(h / 2-\widetilde{\tau}) A_{n}}\right\|_{X_{\beta} \leftarrow X}\left\|F_{n}^{\prime \prime}\left(t_{n}+\sigma \widetilde{\tau}\right)\right\|_{X, \infty} \mathrm{d} \sigma \mathrm{d} \widetilde{\tau} \\
& \leq C h^{2-\beta+\vartheta}\left\|F^{\prime}\right\|_{X_{\vartheta}, \infty}+C h^{3-\beta}\left\|F^{\prime \prime}\right\|_{X, \infty} .
\end{aligned}
$$

Therefore, under the assumptions of the theorem it follows that

$$
\begin{aligned}
\left\|e_{n}\right\|_{X_{\beta}} \leq & \left\|e_{n}^{(0)}\right\|_{X_{\beta}}+\left\|e_{n}^{(1)}\right\|_{X_{\beta}} \\
\leq & C\left\|e_{0}\right\|_{X_{\beta}}+C h \sum_{j=0}^{n-1}\left(t_{n}-t_{j}\right)^{-\beta+\vartheta}\left\|e_{j}\right\|_{X_{\beta}} \\
& +C\left(h^{1+\alpha}(1+|\log h|)\left\|f^{\prime}\right\|_{X_{\vartheta}, \infty}\right. \\
& \left.+h^{2-\beta+\vartheta}\left\|F^{\prime}\right\|_{X_{\vartheta}, \infty}+h^{2}\left\|f^{\prime \prime}\right\|_{X, \infty}+h^{3-\beta}\left\|F^{\prime \prime}\right\|_{X, \infty}\right) \\
& \times h \sum_{j=0}^{n-2}\left(t_{n}-t_{j+1}\right)^{-\beta+\vartheta} \\
& +C h^{2-\beta+\vartheta} h \sum_{j=0}^{n-2}\left(t_{n}-t_{j+1}\right)^{-1}\left\|f^{\prime}\right\|_{X_{\vartheta}, \infty}
\end{aligned}
$$


As a consequence, by interpreting the sums as Riemann sums and estimating them by the corresponding integrals we get

$$
\begin{aligned}
\left\|e_{n}\right\|_{X_{\beta}} \leq C & \left\|e_{0}\right\|_{X_{\beta}}+C h \sum_{j=0}^{n-1}\left(t_{n}-t_{j}\right)^{-\beta+\vartheta}\left\|e_{j}\right\|_{X_{\beta}} \\
+C & \min \left\{h^{1+\alpha}, h^{2-\beta+\vartheta}\right\}(1+|\log h|)\left\|f^{\prime}\right\|_{X_{\vartheta}, \infty} \\
+ & C h^{2-\beta+\vartheta}\left\|F^{\prime}\right\|_{X_{\vartheta}, \infty}+C h^{2}\left\|f^{\prime \prime}\right\|_{X, \infty}+C h^{3-\beta}\left\|F^{\prime \prime}\right\|_{X, \infty} .
\end{aligned}
$$

Finally, the application of a Gronwall lemma shows

$$
\begin{aligned}
\left\|e_{n}\right\|_{X_{\beta}} \leq C & \left\|e_{0}\right\|_{X_{\beta}}+C \min \left\{h^{1+\alpha}, h^{2-\beta+\vartheta}\right\}(1+|\log h|)\left\|f^{\prime}\right\|_{X_{\vartheta}, \infty} \\
& +C h^{2-\beta+\vartheta}\left\|F^{\prime}\right\|_{X_{\vartheta}, \infty}+C h^{2}\left\|f^{\prime \prime}\right\|_{X, \infty}+C h^{3-\beta}\left\|F^{\prime \prime}\right\|_{X, \infty}
\end{aligned}
$$

see also the proof of Lemma 4.4 .

We note that the exponent $\alpha$ in the bound (5.11) as it is restricted by the condition $0<\alpha<\beta-\zeta$ with $\gamma<\zeta<\beta$ is possibly close to 0 . However, regarding the numerical experiments of Section 6 it is essential to raise the size of $\alpha$. For that purpose, let $u$ denote the exact solution of (1.1) started at the numerical initial value $u_{0} \in X_{\beta}$ and assume that it is Lipschitz continuous on $X_{\beta}$; i.e.,

$$
\left\|u\left(t_{n}\right)-u\left(t_{m}\right)\right\|_{X_{\beta}} \leq C\left(t_{n}-t_{m}\right) .
$$

In particular, this relation holds true if the first derivative $u^{\prime}$ is bounded in $X_{\beta}$. Consequently, due to the convergence estimate (5.11) which implies that the order of the numerical scheme in $X_{\beta}$ is at least one, we have

$$
\begin{aligned}
\left\|u_{n}-u_{m}\right\|_{X_{\beta}} & \leq\left\|u_{n}-u\left(t_{n}\right)\right\|_{X_{\beta}}+\left\|u_{m}-u\left(t_{m}\right)\right\|_{X_{\beta}}+\left\|u\left(t_{n}\right)-u\left(t_{m}\right)\right\|_{X_{\beta}} \\
& \leq C h+C\left(t_{n}-t_{m}\right) \leq C\left(t_{n}-t_{m}\right), \quad 0 \leq t_{m} \leq t_{n} \leq T .
\end{aligned}
$$

Altogether, these considerations show that we may set $\alpha=1$ in (5.11) which proves the desired result.

For the proof of the above convergence estimate, the following stability result is needed. Recall the abbreviation $\chi_{m 1}=\chi\left(h A_{m 1}\right)$.

Lemma 5.2. Assume that Hypothesis 2.1 is valid with $\vartheta>0$. Then, the discrete evolution operator $\mathcal{E}_{m}^{n}$ defined in (5.1) fulfills the estimates

$$
\begin{aligned}
\left\|\mathcal{E}_{m}^{n}\right\|_{X_{\beta} \leftarrow X_{\beta}} & +\left\|\left(t_{n+1}-t_{m}\right)^{\beta-\vartheta} \mathcal{E}_{m}^{n}\right\|_{X_{\beta} \leftarrow X_{\vartheta}} \leq C, \\
\left\|\mathcal{E}_{m}^{n} A_{m 1} \chi_{m 1}\right\|_{X_{\beta} \leftarrow X_{\vartheta}} \leq & C h^{-1+\alpha}(1+|\log h|)\left(t_{n+1}-t_{m}\right)^{-\beta+\vartheta} \\
& +C h^{-\beta+\vartheta}\left(t_{n+1}-t_{m}\right)^{-1}, \quad 0 \leq t_{m} \leq t_{n} \leq T,
\end{aligned}
$$

with constant $C>0$ not depending on $n$ and $h$.

Proof. The first estimate of Lemma 5.2 is a direct consequence of Lemma 4.4 For proving the second bound, we correlate the discrete evolution operator with the analytic semigroup generated by $A_{m 1}$. That is, similarly as in the proof of Lemma 4.4, we make use of the identity

$$
\begin{aligned}
& \mathcal{E}_{m}^{n} A_{m 1} \chi_{m 1}=\Delta_{m}^{n} A_{m 1} \chi_{m 1}+A_{m 1} \mathrm{e}^{\left(t_{n+1}-t_{m}\right) A_{m 1}} \chi_{m 1} \\
& =\sum_{j=m+1}^{n-1} \Delta_{j+1}^{n} \widetilde{\Xi}_{j m}+\sum_{j=m+1}^{n} \mathrm{e}^{\left(t_{n+1}-t_{j+1}\right) A_{m 1}} \widetilde{\Xi}_{j m}+A_{m 1} \mathrm{e}^{\left(t_{n+1}-t_{m}\right) A_{m 1}} \chi_{m 1},
\end{aligned}
$$


where $\Delta_{m}^{n}=\mathcal{E}_{m}^{n}-\mathrm{e}^{\left(t_{n+1}-t_{m}\right) A_{m 1}}$ and

$$
\widetilde{\Xi}_{j m}=\left(\mathrm{e}^{h A_{j 1}}-\mathrm{e}^{h A_{m 1}}\right) A_{m 1} \mathrm{e}^{\left(t_{j}-t_{m}\right) A_{m 1}} \chi_{m 1}, \quad j>m .
$$

By means of the integral formula of Cauchy, we obtain the relations

$$
\left\|\widetilde{\Xi}_{j m}\right\|_{X_{\vartheta} \leftarrow X_{\vartheta}} \leq C h\left(t_{j}-t_{m}\right)^{-2+\alpha}, \quad\left\|\widetilde{\Xi}_{n m}\right\|_{X_{\beta} \leftarrow X_{\vartheta}} \leq C h^{1-\beta+\vartheta}\left(t_{n}-t_{m}\right)^{-2+\alpha} \text {. }
$$

Consequently, with the help of the estimate

$$
\left\|A_{m 1} \mathrm{e}^{\left(t_{n+1}-t_{m}\right) A_{m 1}} \chi_{m 1}\right\|_{X_{\beta} \leftarrow X_{\vartheta}} \leq M\left(t_{n+1}-t_{m}\right)^{-1-\beta+\vartheta}
$$

(see also (4.5) and (2.8b) ) and (4.7), we obtain

$$
\begin{aligned}
& \| \mathcal{E}_{m}^{n} A_{m 1} \chi_{m 1}\left\|_{X_{\beta} \leftarrow X_{\vartheta}} \leq \sum_{j=m+1}^{n-1}\right\| \Delta_{j+1}^{n}\left\|_{X_{\beta} \leftarrow X_{\vartheta}}\right\| \widetilde{\Xi}_{j m} \|_{X_{\vartheta} \leftarrow X_{\vartheta}} \\
&+\sum_{j=m+1}^{n-1}\left\|\mathrm{e}^{\left(t_{n+1}-t_{j+1}\right) A_{m 1}}\right\|_{X_{\beta} \leftarrow X_{\vartheta}}\left\|\widetilde{\Xi}_{j m}\right\|_{X_{\vartheta} \leftarrow X_{\vartheta}}+\left\|\widetilde{\Xi}_{n m}\right\|_{X_{\beta} \leftarrow X_{\vartheta}} \\
& \quad+\left\|A_{m 1} \mathrm{e}^{\left(t_{n+1}-t_{m}\right) A_{m 1}} \chi_{m 1}\right\|_{X_{\beta} \leftarrow X_{\vartheta}} \\
& \leq C h \sum_{j=m+1}^{n-1}\left(t_{n+1}-t_{j+1}\right)^{-\beta+\vartheta+\alpha}\left(t_{j}-t_{m}\right)^{-2+\alpha}+C\left(t_{n+1}-t_{m}\right)^{-1-\beta+\vartheta} \\
& \leq C h^{-1+\alpha}(1+|\log h|)\left(t_{n+1}-t_{m}\right)^{-\beta+\vartheta}+C\left(t_{n+1}-t_{m}\right)^{-1-\beta+\vartheta}
\end{aligned}
$$

which yields the specified estimate.

\section{EXTENSION AND NUMERICAL EXAMPLE}

In this section, we discuss a possible extension of the Magnus-type integrator (3.1) to quasi-linear equations with an additional inhomogeneity and illustrate the theoretical convergence result by a numerical example. Throughout, we employ the hypotheses and notation introduced in Sections 25 .

6.1. Extension to inhomogeneous quasi-linear problems. The convergence analysis of Section 5 easily generalises to problems with an additional inhomogeneous part. In view of our numerical example, we consider an abstract initial value problem of the form

$$
u^{\prime}(t)=A(u(t)) u(t)+b(t), \quad 0<t \leq T, \quad u(0) \text { given, }
$$

involving a time-dependent map $b:[0, T] \rightarrow X$. In this case, the numerical method (3.1) for the quasi-linear parabolic equation (1.1) is modified as follows:

$$
\begin{gathered}
U_{n 1}=\mathrm{e}^{h / 2 A_{n}} u_{n}+h / 2 \varphi\left(h / 2 A_{n}\right) b_{n}, \quad b_{n}=b\left(t_{n}\right), \\
u_{n+1}=\mathrm{e}^{h A_{n 1}} u_{n}+h \varphi\left(h A_{n 1}\right) b_{n 1}, \quad b_{n 1}=b\left(t_{n 1}\right), \quad n \geq 0
\end{gathered}
$$

(see (2.8a)). Similar considerations as in Section 5 show that the following convergence result is valid with maps $\widetilde{f}_{n}$ and $\widetilde{F}_{n}$ defined by

$$
\widetilde{f}_{n}(t)=f_{n}(t)+b(t)-b_{n 1}, \quad \widetilde{F}_{n}(t)=F_{n}(t)+b(t)-b_{n}, \quad n \geq 0,
$$

provided that first and second derivatives of $b$ are bounded in certain intermediate spaces; see also (5.4) and (5.8). 
Theorem 6.1 (Convergence). Assume that the requirements of Theorem [5.1] are satisfied. Then, for $h>0$ chosen sufficiently small, the numerical method (6.2) applied to the abstract initial value problem (6.1) fulfills the convergence bound

$$
\begin{aligned}
\left\|u_{n}-u\left(t_{n}\right)\right\|_{X_{\beta}} \leq C & \left\|u_{0}-u(0)\right\|_{X_{\beta}} \\
+ & C h^{2-\beta+\vartheta}\left((1+|\log h|)\left\|\widetilde{f}^{\prime}\right\|_{X_{\vartheta}, \infty}+\left\|\widetilde{F}^{\prime}\right\|_{X_{\vartheta}, \infty}\right) \\
& \quad+C h^{2}\left(\left\|\widetilde{f}^{\prime \prime}\right\|_{X, \infty}+h^{1-\beta}\left\|\widetilde{F}^{\prime \prime}\right\|_{X, \infty}\right), \quad 0 \leq t_{n} \leq T,
\end{aligned}
$$

with constant $C>0$ not depending on $n$ and $h$.

6.2. Numerical example. The following application illustrates the above convergence result. In order to keep the realisation simple, we restrict ourselves to a parabolic initial boundary value problem in one space dimension.

Example 6.2. We consider a one-dimensional initial boundary value problem for a function $U:[0,1]^{2} \rightarrow \mathbb{R}:(x, t) \rightarrow U(x, t)$ comprising a quasi-linear partial differential equation with the additional inhogoneneous part

$$
\partial_{t} U(x, t)=\mathcal{A}(U(x, t)) U(x, t)+B(x, t), \quad 0<x \leq 1, \quad 0<t \leq 1,
$$

subject to a homogeneous Dirichlet boundary condition and an initial condition

$$
U(0, t)=0=U(1, t), \quad 0 \leq t \leq 1, \quad U(x, 0)=U_{0}(x), \quad 0 \leq x \leq 1 .
$$

For functions $v \in C^{1}(0,1)$ and $w \in C^{2}(0,1)$ the differential operator $\mathcal{A}$ is given by

$$
\mathcal{A}(v(x)) w(x)=a\left(x, v(x), \partial_{x} v(x)\right) \partial_{x}^{2} w(x), \quad 0<x \leq 1,
$$

with coefficient $a: \mathbb{R}^{3} \rightarrow \mathbb{R}$ satisfying suitable regularity and boundedness assumptions. Specifically, for the numerical example we set

$$
a(x, p, q)=1+p^{2}+c q^{2}, \quad c=0,1,
$$

and determine the function $B$ and the initial condition $U_{0}$ such that the exact solution of (6.4) is given by $U(x, t)=\mathrm{e}^{-t} x(1-x)$. Note that $U$ fulfills the homogeneous Dirichlet boundary condition.

We let $(u(t))(x)=U(x, t),(A(v) w)(x)=\mathcal{A}(v(x)) w(x)$, and $(b(t))(x)=B(x, t)$. With this notation, the initial boundary value problem (6.4) takes the form of an initial value problem (6.1) on the Banach space $X=L^{p}(\Omega)$ for $1<p<\infty$ with domain of $A(v)$ given by the function space $D=W^{2, p}(0,1) \cap W_{0}^{1, p}(0,1)$. From Example 2.3 we thus conclude that the linear operator family $A: X_{\gamma} \rightarrow$ $L\left(X_{1+\vartheta}, X_{\vartheta}\right)$ satisfies Hypothesis 2.1 with $\vartheta=0$ and constant $\gamma$ restricted by the condition $c / 2+(2 p)^{-1}<\gamma<1$. Furthermore, due to the fact that the domain of $A(v)^{2}$ equals

$$
D^{2}=D\left(A(v)^{2}\right)=\left\{w \in W^{4, p}(0,1) \cap W_{0}^{1, p}(0,1):\left.\partial_{x}^{2} w(x)\right|_{x=0,1}=0\right\}
$$

and therefore does not depend on $v \in V$, the same holds true for any intermediate space $D \subset X_{1+\vartheta} \subset D\left(A(v)^{2}\right)$. Besides, $A: X_{\gamma} \rightarrow L\left(D^{2}, D\right)$ is Lipschitz continuous with respect to $v$. As a consequence, Hypothesis 2.1 remains valid for every $0 \leq$ $\vartheta \leq 1$.

In the present situation, all requirements of Theorem 5.1 are fulfilled. Namely, the exact solution $U(x, t)$ and the data $a(x, p, q)$ and $B(x, t)$ are sufficiently regular. Therefore, the maps $\widetilde{f}_{n}$ and $\widetilde{F}_{n}$ defined in (6.3) are twice differentiable in $X$, and, besides, the Fréchet derivative $A^{\prime}(v): X_{\gamma} \rightarrow L\left(X_{1+\vartheta}, X_{\vartheta}\right)$ is bounded. A result 
TABLE 1. Numerically observed temporal convergence order in the discrete $X_{\beta}$-norm for $c=0, p=2, \beta=(2 p)^{-1}=1 / 4$ (left), $\beta=1$ (right). Expected values $\kappa_{1 / 4} \approx 2, \kappa_{1} \approx 1+1 / 4$; see (6.5b).

\begin{tabular}{|c||c|c|c|}
\hline$h \backslash M$ & 50 & 100 & 150 \\
\hline \hline $2^{-2}$ & 1.8988 & 1.8987 & 1.8986 \\
\hline $2^{-3}$ & 1.9021 & 1.9018 & 1.9017 \\
\hline $2^{-4}$ & 1.8965 & 1.8959 & 1.8957 \\
\hline $2^{-5}$ & 1.9078 & 1.9067 & 1.9064 \\
\hline $2^{-6}$ & 1.9184 & 1.9163 & 1.9159 \\
\hline $2^{-7}$ & 1.9291 & 1.9252 & 1.9244 \\
\hline $2^{-8}$ & 1.9409 & 1.9333 & 1.9319 \\
\hline $2^{-9}$ & 1.9553 & 1.9415 & 1.9388 \\
\hline $2^{-10}$ & 1.9728 & 1.9508 & 1.9457 \\
\hline
\end{tabular}

\begin{tabular}{|c||c|c|c|}
\hline$h \backslash M$ & 50 & 100 & 150 \\
\hline \hline $2^{-2}$ & 1.3462 & 1.3335 & 1.3293 \\
\hline $2^{-3}$ & 1.2770 & 1.2621 & 1.2572 \\
\hline $2^{-4}$ & 1.2987 & 1.2760 & 1.2686 \\
\hline $2^{-5}$ & 1.3185 & 1.2847 & 1.2738 \\
\hline $2^{-6}$ & 1.3480 & 1.2977 & 1.2817 \\
\hline $2^{-7}$ & 1.3947 & 1.3181 & 1.2946 \\
\hline $2^{-8}$ & 1.4679 & 1.3495 & 1.3141 \\
\hline $2^{-9}$ & 1.5817 & 1.3977 & 1.3437 \\
\hline $2^{-10}$ & 1.7389 & 1.4730 & 1.3889 \\
\hline
\end{tabular}

in Grisvard 14 that characterises the intermediate spaces $X \subset X_{\vartheta} \subset D$ implies that any function which is spatially smooth but does not satisfy further boundary conditions belongs to $X_{\vartheta}$ as long as $\vartheta<(2 p)^{-1}$; see also the discussion in [11. That is, the first derivatives of $\widetilde{f}_{n}$ and $\widetilde{F}_{n}$ are bounded in $X_{\vartheta}$ for $\vartheta<(2 p)^{-1}$. Moreover, the exact solution of (6.4) lies in the intermediate space $X_{1+\vartheta}$ if $\vartheta<(2 p)^{-1}$ and its first time derivative $\partial_{t} U(x, t)=-U(x, t)$ remains bounded in $X_{\beta}$ for arbitrary $0 \leq \beta \leq 1$. As a consequence, according to Theorem 5.1, the expected convergence order with respect to the norm of the Sobolev space $X_{\beta}$ is

$$
\kappa_{\beta}=2-\beta+\vartheta, \quad c / 2+(2 p)^{-1}<\gamma<1, \quad \vartheta<(2 p)^{-1},
$$

where $\gamma<\beta<1$.

For the numerical example, the partial differential equation is discretised in space by symmetric finite differences of grid length $\Delta x=(M+2)^{-1}$, and, for the time integration, we apply the numerical method (6.2) with stepsize $h>0$. The numerical temporal order of convergence measured in the discrete $X_{\beta}$-norm is determined

TABLE 2. Numerically observed temporal convergence order in the discrete $X_{\beta}$-norm for $c=0, p=100, \beta=(2 p)^{-1}=1 / 200$ (left), $\beta=1$ (right). Expected values $\kappa_{1 / 200} \approx 2, \kappa_{1} \approx 1+1 / 200$; see $(\underline{6.5 \mathrm{~b}})$.

\begin{tabular}{|c||c|c|c|}
\hline$h \backslash M$ & 50 & 100 & 150 \\
\hline \hline $2^{-2}$ & 2.0180 & 2.0180 & 2.0180 \\
\hline $2^{-3}$ & 2.0465 & 2.0464 & 2.0463 \\
\hline $2^{-4}$ & 1.9818 & 1.9813 & 1.9812 \\
\hline $2^{-5}$ & 1.9827 & 1.9819 & 1.9817 \\
\hline $2^{-6}$ & 1.9859 & 1.9843 & 1.9840 \\
\hline $2^{-7}$ & 1.9910 & 1.9880 & 1.9874 \\
\hline $2^{-8}$ & 1.9968 & 1.9920 & 1.9909 \\
\hline $2^{-9}$ & 2.0001 & 1.9965 & 1.9943 \\
\hline $2^{-10}$ & 2.0137 & 2.0012 & 1.9978 \\
\hline
\end{tabular}

\begin{tabular}{|c||c|c|c|}
\hline$h \backslash M$ & 50 & 100 & 150 \\
\hline \hline $2^{-2}$ & 1.0854 & 1.0661 & 1.0601 \\
\hline $2^{-3}$ & 1.0752 & 1.0492 & 1.0408 \\
\hline $2^{-4}$ & 1.0895 & 1.0504 & 1.0375 \\
\hline $2^{-5}$ & 1.1184 & 1.0616 & 1.0429 \\
\hline $2^{-6}$ & 1.1662 & 1.0831 & 1.0560 \\
\hline $2^{-7}$ & 1.2396 & 1.1169 & 1.0775 \\
\hline $2^{-8}$ & 1.3500 & 1.1678 & 1.1101 \\
\hline $2^{-9}$ & 1.5106 & 1.2439 & 1.1584 \\
\hline $2^{-10}$ & 1.7118 & 1.3572 & 1.2302 \\
\hline
\end{tabular}


TABLE 3. Numerically observed temporal convergence order in the discrete $X_{\beta}$-norm for $c=1, p=2, \beta=1 / 2+(2 p)^{-1}=3 / 4$ (left), $\beta=1$ (right). Expected values $\kappa_{3 / 4} \approx 1+1 / 2, \kappa_{1} \approx 1+1 / 4$; see $6.5 \mathrm{c}$.

\begin{tabular}{|c||c|c|c|}
\hline$h \backslash M$ & 50 & 100 & 150 \\
\hline \hline $2^{-2}$ & 1.5985 & 1.5955 & 1.5948 \\
\hline $2^{-3}$ & 1.4579 & 1.4533 & 1.4523 \\
\hline $2^{-4}$ & 1.4644 & 1.4568 & 1.4550 \\
\hline $2^{-5}$ & 1.4922 & 1.4788 & 1.4756 \\
\hline $2^{-6}$ & 1.5154 & 1.4920 & 1.4863 \\
\hline $2^{-7}$ & 1.5474 & 1.5067 & 1.4968 \\
\hline $2^{-8}$ & 1.5963 & 1.5263 & 1.5090 \\
\hline $2^{-9}$ & 1.6737 & 1.5560 & 1.5261 \\
\hline $2^{-10}$ & 1.7854 & 1.6040 & 1.5528 \\
\hline
\end{tabular}

\begin{tabular}{|c||c|c|c|}
\hline$h \backslash M$ & 50 & 100 & 150 \\
\hline \hline $2^{-2}$ & 1.2614 & 1.2482 & 1.2438 \\
\hline $2^{-3}$ & 1.2056 & 1.1915 & 1.1868 \\
\hline $2^{-4}$ & 1.2529 & 1.2315 & 1.2244 \\
\hline $2^{-5}$ & 1.2864 & 1.2546 & 1.2443 \\
\hline $2^{-6}$ & 1.3222 & 1.2748 & 1.2599 \\
\hline $2^{-7}$ & 1.3712 & 1.2995 & 1.2775 \\
\hline $2^{-8}$ & 1.4432 & 1.3326 & 1.2997 \\
\hline $2^{-9}$ & 1.5524 & 1.3802 & 1.3301 \\
\hline $2^{-10}$ & 1.7069 & 1.4520 & 1.3741 \\
\hline
\end{tabular}

from the numerical and exact solution values. In particular, if the differential operator involves no first derivative (i.e., $c=0$ in (6.4d) ) for the limiting cases $\beta=(2 p)^{-1}$ and $\beta=1$, we expect a numerical convergence order of approximately

$$
\kappa_{(2 p)^{-1}}=2-(2 p)^{-1}+\vartheta \approx 2, \quad \kappa_{1}=1+\vartheta \approx 1+(2 p)^{-1} ;
$$

see (6.5a). On the other hand, for the case where $c=1$, we have

$$
\text { (6.5c) } \kappa_{1 / 2+(2 p)^{-1}}=1+1 / 2-(2 p)^{-1}+\vartheta \approx 1+1 / 2, \quad \kappa_{1}=1+\vartheta \approx 1+(2 p)^{-1} .
$$

The results of the numerical experiment for $p=2$ and $p=100$ are displayed in Tables 14. The numbers observed are in good agreement with the expected values. We remark that for the chosen values of $M$ and $h$ the problem becomes nonstiff as the temporal stepsize $h$ tends to $2^{-10}$. Thus, the numerical order approaches the classical convergence order 2 .

TABLE 4. Numerically observed temporal convergence order in the discrete $X_{\beta}$-norm for $c=1, p=100, \beta=1 / 2+(2 p)^{-1}=1 / 2+$ $1 / 200$ (left), $\beta=1$ (right). Expected values $\kappa_{1 / 2+1 / 200} \approx 1+1 / 2$, $\kappa_{1} \approx 1+1 / 200$; see (6.5c).

\begin{tabular}{|c||c|c|c|}
\hline$h \backslash M$ & 50 & 100 & 150 \\
\hline \hline $2^{-2}$ & 1.6447 & 1.6441 & 1.6440 \\
\hline $2^{-3}$ & 1.4681 & 1.4669 & 1.4667 \\
\hline $2^{-4}$ & 1.4697 & 1.4677 & 1.4673 \\
\hline $2^{-5}$ & 1.4835 & 1.4791 & 1.4784 \\
\hline $2^{-6}$ & 1.4858 & 1.4865 & 1.4849 \\
\hline $2^{-7}$ & 1.5559 & 1.4946 & 1.4904 \\
\hline $2^{-8}$ & 1.4690 & 1.4935 & 1.4951 \\
\hline $2^{-9}$ & 1.5942 & 1.5602 & 1.4983 \\
\hline $2^{-10}$ & 1.7540 & 1.4754 & 1.5582 \\
\hline
\end{tabular}

\begin{tabular}{|c||c|c|c|}
\hline$h \backslash M$ & 50 & 100 & 150 \\
\hline \hline $2^{-2}$ & 0.9801 & 0.9598 & 0.9535 \\
\hline $2^{-3}$ & 1.0091 & 0.9838 & 0.9757 \\
\hline $2^{-4}$ & 1.0474 & 1.0102 & 0.9979 \\
\hline $2^{-5}$ & 1.0875 & 1.0338 & 1.0161 \\
\hline $2^{-6}$ & 1.1397 & 1.0613 & 1.0358 \\
\hline $2^{-7}$ & 1.2132 & 1.0979 & 1.0609 \\
\hline $2^{-8}$ & 1.3199 & 1.1488 & 1.0947 \\
\hline $2^{-9}$ & 1.4742 & 1.2222 & 1.1423 \\
\hline $2^{-10}$ & 1.6743 & 1.3298 & 1.2109 \\
\hline
\end{tabular}




\section{ACKNOWLEDGMENTS}

The authors are grateful to Alexander Ostermann for several discussions related to this work and valuable comments on the manuscript. The work of Mechthild Thalhammer was supported by Fonds zur Förderung der wissenschaftlichen Forschung (FWF) under project H210-N13. The research stays of Mechthild Thalhammer in Valladolid in June 2004 and of Césareo González in Innsbruck in October 2004 were supported by DGI-MCYT under grant BFM2001-2013/2138 cofinanced by FEDER funds and by JCYL under grant VA112/02.

\section{REFERENCES}

1. H. Amann, Quasilinear evolution equations and parabolic systems. Trans. Amer. Math. Soc. 293 (1986) 191-227. MR0814920 (87d:35070)

2. H. Amann, Dynamic theory of quasilinear parabolic equations - I. Abstract evolution equations. Nonlin. Anal. Th. Meth. and Appl. 12 (1988) 895-919. MR0960634 (89j:35072)

3. H. Amann, Highly degenerate quasilinear parabolic systems. Ann. Scuola Norm. Sup. Pisa Ser. IV 18 (1991) 135-166. MR:1118224(92m:35145)

4. H. Amann, Linear and Quasilinear Parabolic Problems. Vol. I Abstract Theory. In: Monographs in Mathematics Vol. 89, Birkhäuser, Basel, 1995. MR.1345385 (96g:34088)

5. N. Aparicio, S. Malham, and M. Oliver, Numerical evaluation of the Evans function by Magnus integration (2004). BIT 45 (2005) 219-258. MR2176193

6. J. Bergh and J. Löfström, Interpolation Spaces. An Introduction. Springer, Berlin, 1976. MR0482275 (58:2349)

7. S. Blanes, F. CASAS, And J. Ros, Improved high order integrators based on the Magnus expansion. BIT 40 (2000) 434-450. MR1780401 (2001f:65078)

8. H. Brunnner And P.J. VAn Der HouWen, The numerical solution of Volterra equations. CWI Monographs 3. North-Holland, Amsterdam, 1986. MR0871871 (88g:65136)

9. PH. Clément, C. J. van Duijn, and Shuanhu Li, On a nonlinear elliptic-parabolic partial differential equation system in a two-dimensional groundwater flow problem. SIAM J. Math. Anal. 23/4 (1992) 836-851. MR1166560 (93h:35089)

10. J. VAN Den EShOF AND M. HochBRUCK, Preconditioning Lanczos approximations to the matrix exponential (2004). To appear in SIAM J. Sci. Comp.

11. C. González, A. Ostermann, and M. Thalhammer, A second-order Magnus integrator for non-autonomous parabolic problems (2004). J. Comp. Appl. Math. (in press).

12. C. GonzÁlez And C. Palencia, Stability of time-stepping methods for time-dependent parabolic problems: the Hölder case. Math. Comp. 68 (1999) 73-89. MR.1609666 (99c:65108)

13. C. González and C. Palencia, Stability of Runge-Kutta methods for quasilinear parabolic problems. Math. Comp. 69 (2000) 609-628. MR.1659851 (2000i:65130)

14. P. Grisvard, Caractérisation de quelques espaces d'interpolation. Arch. Rat. Mech. Anal. 25 (1967) 40-63. MR0213864 (35:4718)

15. D. Henry, Geometric Theory of Semilinear Parabolic Equations. Lecture Notes in Mathematics 840, Springer, Berlin, 1981. MR0610244 (83j:35084)

16. M. Hochbruck AND CH. Lubich, On Krylov subspace approximations to the matrix exponential operator. SIAM J. Numer. Anal. 34 (1997) 1911-1925. MR1472203 (98h:65018)

17. M. Hochbruck AND CH. Lubich, On Magnus integrators for time-dependent Schrödinger equations. SIAM J. Numer. Anal. 41 (2003) 945-963. MR2005189 (2004g:65089)

18. A. Iserles And S.P. NøRSETt, On the solution of linear differential equations in Lie groups. Phil. Trans. R. Soc. Lond. A 357 (1999) 983-1019. MR.1694700 (2000d:34022)

19. CH. LuBich, Integrators for Quantum Dynamics: A Numerical Analyst's Brief Review. In: Quantum Simulations of Complex Many-Body Systems: From Theory to Algorithms, Lecture Notes, J. Grotendorst, D. Mary, A. Muramatsu (Eds.), John von Neumann Institute for Computing, Jülich, NIC Series 10 (2002) 459-466.

20. A. Lunardi, Analytic Semigroups and Optimal Regularity in Parabolic Problems. Birkhäuser, Basel, 1995. MR1329547(96e:47039)

21. W. Magnus, On the exponential solution of a differential equation for a linear operator. Comm. Pure Appl. Math. 7 (1954) 649-673. MR0067873 (16:790a) 
22. C. Moler And Ch. VAn Loan, Nineteen dubious ways to compute the exponential of a matrix, twenty-five years later. SIAM Rev. 45 no. 1 (2003) 3-49. MR.1981253 (2004d:15012)

23. H. Munthe-KaAs And B. Owren, Computations in a free Lie algebra. Phil. Trans. R. Soc. Lond. A 357 (1999) 957-981. MR1694699 (2000f:17005)

24. A. Ostermann and M. Thalhammer, Non-smooth data error estimates for linearly implicit Runge-Kutta methods. IMA J. Numer. Anal. 20 (2000) 167-184. MR1752261(2001a:65085)

25. A. PAZY, Semigroups of Linear Operators and Applications to Partial Differential Equations. Springer, New York, 1983. MR0710486 (85g:47061)

26. M. Thalhammer, A second-order Magnus type integrator for non-autonomous semilinear parabolic problems (2004). Submitted to IMA J. Numer. Anal.

27. M. Thalhammer, A fourth-order commutator-free exponential integrator for non-autonomous differential equations (2005). Submitted to SIAM J. Numer. Anal.

28. H. TRIEBEL, Interpolation Theory, Function Spaces, Differential Operators. North-Holland, Amsterdam, 1978. MR0500580 (80i:46032a)

29. J. Wensch, M. DÄne, W. Hergert, AND A. ERnst, The solution of stationary ODE problems in quantum mechanics by Magnus methods with stepsize control. Comp. Phys. Comm. 160 (2004) 129-139.

Departamento de Matemática Aplicada y Computación, Facultad de Ciencias, UniVERSidAd de VAlladolid, E-47011 Valladolid, Spain

E-mail address: cesareo@mac.cie.uva.es

Institut für Mathematik, FakUltät Für Mathematik, Informatik Und Physik, UniverSitÄt Innsbruck, Technikerstrasse 25/7, A-6020 Innsbruck, Austria

E-mail address: Mechthild.Thalhammer@uibk.ac.at 